\title{
STOCHASTIC SWITCHING IN INFINITE DIMENSIONS WITH APPLICATIONS TO RANDOM PARABOLIC PDES
}

\author{
SEAN D. LAWLEY, JONATHAN C. MATTINGLY, AND MICHAEL C. REED
}

\begin{abstract}
We consider parabolic PDEs with randomly switching boundary conditions. In order to analyze these random PDEs, we consider more general stochastic hybrid systems and prove convergence to, and properties of, a stationary distribution. Applying these general results to the heat equation with randomly switching boundary conditions, we find explicit formulae for various statistics of the solution and obtain almost sure results about its regularity and structure. These results are of particular interest for biological applications as well as for their significant departure from behavior seen in PDEs forced by disparate Gaussian noise. Our general results also have applications to other types of stochastic hybrid systems, such as ODEs with randomly switching right-hand sides.
\end{abstract}

keywords. Random PDEs, hybrid dynamical systems, switched dynamical systems, piecewise deterministic Markov process, ergodicity.

AMS subject classifications. 35R60, 37H99, 46N20, 60H15, 92C30

\section{INTRODUCTION}

The primary motivation for this paper is to study parabolic partial differential equations (PDEs) with randomly switching boundary conditions. More precisely, given an elliptic differential operator, $L$, on a domain $D \subset \mathbb{R}^{d}$, we want to study the stochastic process $u(t, x)$ that solves $\partial_{t} u=L u$ in $D$ subject to boundary conditions that switch at random times between two given boundary conditions.

This type of random PDE is an example of a stochastic hybrid system. The word "hybrid" is used because these stochastic processes involve both continuous dynamics and discrete events. In this example, the continuous dynamics are the different boundary value problems corresponding to the different boundary conditions for the given PDE, and the discrete events are when the boundary condition switches.

In general, a stochastic hybrid system is a continuous-time stochastic process with two components: a continuous component $\left(X_{t}\right)_{t \geq 0}$ and a jump component $\left(J_{t}\right)_{t \geq 0}$. The jump component, $J_{t}$, is a jump process on a finite set, and for each element of its state space we assign some continuous dynamics to $X_{t}$. In between jumps of $J_{t}$, the component $X_{t}$ evolves according to the dynamics associated with the current state of $J_{t}$. When $J_{t}$ jumps, the component $X_{t}$ switches to following the dynamics associated with the new state of $J_{t}$.

An ordinary differential equation (ODE) with a switching right-hand side is the type of stochastic hybrid system that is most commonly used in applications.

Department of Mathematics, Duke University, Box 90320, Durham, NC 27708-0320 USA, \{lawley@math.duke.edu, jonm@math.duke.edu, reed@math.duke.edu\}. 
Such ODE switching systems have been used extensively in applied areas such as control theory, computer science, and engineering (for example, 42, [8, [3], and [28]). More recently, these systems have been used in diverse areas of biology (for example, molecular biology [10, 34, 9], ecology [43, and epidemiology [20]). Furthermore, such ODE switching systems have also recently been the subject of much mathematical study ([26], 12], 6], [5], 2], 21], 22], and [4]).

Comparatively, stochastic hybrid systems stemming from PDEs have received little attention. While deterministic PDEs coupled to random boundary conditions have been studied, the random boundary conditions have typically been assumed to involve some Gaussian noise forcing ([1, [17, [38, [37, and [15]). The randomness enters our PDE system in a fundamentally different way than in Stochastic PDEs which are driven by additive space-time white noise (or even spatially smoother Gaussian fields). There the fine scales are often asymptotically independent of each other [32, 33]. Here, there is a single piece of randomness which dictates the fine structure and hence the fine scales, though not asymptotically deterministic, are asymptotically perfectly correlated. (See Proposition 6 for more details.)

We were led to study such random PDEs by various biological applications. One application is to insect respiration. Essentially all insects breathe via a network of tubes that allow oxygen and carbon dioxide to diffuse to and from their cells 40. Air enters and exits this network through valves (called spiracles) in the exoskeleton, which regulate air flow by opening and closing. Surprisingly, spiracles open and close quite irregularly in time, and insect physiologists have proposed at least five hypotheses to explain this behavior [11. In order to address these competing hypotheses, physiologists would like to understand how much cellular oxygen uptake decreases as a result of the spiracles closing. To answer this question, we model the oxygen concentration in one of the respiratory tubes. As diffusion is the primary mechanism for oxygen movement in the tubes ([29]), the oxygen concentration satisfies the heat equation and the opening and closing of the spiracle corresponds to a randomly switching boundary condition. Our analysis of this model has some surprising implications for this physiological application.

Our paper is organized as follows. In Section 2, we consider more general stochastic hybrid systems from the viewpoint of iterated random functions (see [16] or 24, 25] for a review of iterated random functions). Assuming that the continuous dynamics are contracting on average, we prove convergence to a stationary distribution and describe the structure and properties of this distribution. In Section 3 , we apply these general results to the random PDE problems described above. We show that the mean of the process satisfies the PDE and that the mean of the stationary distribution satisfies the time homogeneous version of the PDE. Then in Section 4, we apply our results from Sections 2 and 3 to the one-dimensional heat equation with randomly switching boundary conditions. We find explicit formulae for various statistics of the solution and obtain almost sure results about its regularity and structure. There, we also show that our general results have applications to other types of stochastic hybrid systems, such as ODEs with randomly switching right-hand sides. Finally, we end Section 4 by explaining that our results can be applied to the question in insect physiology mentioned above.

We conclude this introduction by giving two examples that motivated our study. We return to these examples in Section 4 . Consider the heat equation on the interval $[0, L]$ with an absorbing boundary condition at 0 and a randomly switching 
boundary condition at $L$. Let the switching be controlled by a Markov jump process, $J_{t}$, on $\{0,1\}$ with $r_{0}$ and $r_{1}$ the respective rates for leaving states 0 and 1 . In the following two examples, we consider different possible boundary conditions at $L$.

Example 1. Suppose the boundary condition at $L$ switches between an inhomogeneous Dirichlet condition and a Neumann no flux condition. More precisely, consider the stochastic process $u(t, x) \in L^{2}[0, L]$ that solves

$$
\begin{aligned}
\partial_{t} u & =D \Delta u \quad \text { in }(0, L) \\
u(0, t) & =0 \quad \text { and } \quad J_{t} u_{x}(L, t)+\left(1-J_{t}\right)(u(L, t)-b)=0 .
\end{aligned}
$$

We show in Section 4.1 that as $t \rightarrow \infty$, the process $u(t, x)$ converges in distribution to an $L^{2}[0, L]$-valued random variable whose expectation is a linear function. Letting $\gamma=L \sqrt{\left(r_{0}+r_{1}\right) / D}$ and $\rho=r_{0} / r_{1}$, we will show that the slope of this function is

$$
\left(1+\frac{\rho}{\gamma} \tanh (\gamma)\right)^{-1} \frac{b}{L}
$$

Example 2. Suppose the boundary condition at $L$ switches between an inhomogeneous Dirichlet condition and a homogeneous Dirichlet condition. More precisely, consider the stochastic process $u(t, x) \in L^{2}[0, L]$ that solves

$$
\begin{aligned}
\partial_{t} u & =D \Delta u \quad \text { in }(0, L) \\
u(0, t) & =0 \quad \text { and } \quad J_{t} u(L, t)+\left(1-J_{t}\right)(u(L, t)-b)=0 .
\end{aligned}
$$

We show in Section 4.2 that as $t \rightarrow \infty$, the process $u(t, x)$ converges in distribution to an $L^{2}[0, L]$-valued random variable whose expectation is a linear function. Letting $p=r_{0} /\left(r_{0}+r_{1}\right)$, we will show that the slope of this function is

$$
(1-p) \frac{b}{L}
$$

The expectations for Examples 1 and 2 are quite different. In Example 2, the expectation is the solution to the time homogenous PDE with boundary conditions given by the average of the two possible boundary conditions. We will see in Section 4.2 that this simple result holds because the process switches between boundary conditions of the same type and the corresponding semigroups commute. Moreover, because the boundary conditions are the same type we will be able to compute individual and joint statistics of the Fourier coefficients of the stationary solution and show that this solution almost surely has a very specific structure and regularity.

In both examples, the expectation is a linear function with slope given by $b / L$ multiplied by a factor less than one. While in Example 2 this factor is simply the proportion of time the boundary condition is inhomogeneous, the factor in Example 1 is an unexpected expression involving the hyperbolic tangent. Furthermore, while the factor in Example 1 still depends on the proportion of time the boundary condition is inhomogeneous, it also depends on how often the boundary conditions switch. Observe that if we keep this proportion fixed by fixing the ratio $r_{0} / r_{1}$, and take the frequency of switches small by letting $r_{0}+r_{1}$ go to 0 , then the slope for Example 1 approaches the same slope as in the Example 2 And if we keep the ratio $r_{0} / r_{1}$ fixed, but let the $r_{0}+r_{1}$ go to infinity, then the slope for Example 1 approaches $b / L$. Some biological implications of this result are discussed in Section 4.3 . 


\section{Abstract Setting}

We first consider stochastic hybrid systems in a separable Banach space $X$. Under certain contractivity assumptions, we prove that the process converges in distribution at large time and we show that the limiting distribution satisfies certain invariance properties. Although applicable to a range of stochastic hybrid systems, the contents of this section will prove particularly useful when we consider PDEs with randomly switching boundary conditions in Sections 3 and 4 .

2.1. Discrete-time process. We first define the set $\Omega$ of all possible switching environments and equip it with a probability measure $\mathbb{P}$ and associated expectation $\mathbb{E}$. Let $\mu_{0}$ and $\mu_{1}$ be two probability distributions on the positive real line. Define each switching environment, $\omega \in \Omega$, as the sequence $\omega=\left(\omega_{1}, \omega_{2}, \ldots\right)$, where each $\omega_{k}$ is a pair of non-negative real numbers, $\left(\tau_{0}^{k}, \tau_{1}^{k}\right)$, drawn from $\mu_{0} \times \mu_{1}$. We take $\mathbb{P}$ to be the infinite product measure generated by $\mu_{0} \times \mu_{1}$. To summarize some notation

$$
\omega=\left(\omega_{1}, \omega_{2}, \omega_{3}, \ldots\right)=\left(\left(\tau_{0}^{1}, \tau_{1}^{1}\right),\left(\tau_{0}^{2}, \tau_{1}^{2}\right),\left(\tau_{0}^{3}, \tau_{1}^{3}\right), \ldots\right) \in \Omega .
$$

For each $t \geq 0$, let $\Phi_{t}^{0}(x)$ and $\Phi_{t}^{1}(x)$ be two mappings from a separable Banach space $X$ to itself. Make the following assumptions on $\Phi_{t}^{i}$ for each $i \in\{0,1\}, t \geq 0$, $x, y \in X$, and with $\tau_{i}$ an independent draw from $\mu_{i}$.

(a) $\Phi_{t}^{0}(x)=x=\Phi_{t}^{1}(x)$ if $t=0$

(b) $t \mapsto \Phi_{t}^{i}(x) \in X$ is continuous

(c) $\mathbb{E}\left|\Phi_{\tau_{i}}^{i}(x)\right|<\infty$

(d) $\left|\Phi_{t}^{i}(x)-\Phi_{t}^{i}(y)\right| \leq K_{i}(t)|x-y|$ for some $K_{i}(t)$

(e) $\mathbb{E} K_{0}\left(\tau_{1}\right) \mathbb{E} K_{1}\left(\tau_{1}\right)<1$

For each $\omega \in \Omega, x \in X$, and natural number $k$, define the compositions

$$
G_{\omega}^{k}(x):=\Phi_{\tau_{1}^{k}}^{1} \circ \Phi_{\tau_{0}^{k}}^{0}(x) \quad \text { and } \quad F_{\omega}^{k}(x):=\Phi_{\tau_{0}^{k}}^{0} \circ \Phi_{\tau_{1}^{k}}^{1}(x) .
$$

For each $\omega \in \Omega, x \in X$, and natural number $n>0$, we define the forward maps $\varphi^{n}$ and $\gamma^{n}$, and the backward maps $\varphi^{-n}$ and $\gamma^{-n}$ by the following compositions of $G$ and $F$ :

$$
\begin{aligned}
& \varphi_{\omega}^{n}(x)=G_{\omega}^{n} \circ \cdots \circ G_{\omega}^{1}(x) \quad \text { and } \quad \gamma_{\omega}^{n}(x)=F_{\omega}^{n} \circ \cdots \circ F_{\omega}^{1}(x), \\
& \varphi_{\omega}^{-n}(x)=G_{\omega}^{1} \circ \cdots \circ G_{\omega}^{n}(x) \quad \text { and } \quad \gamma_{\omega}^{-n}(x)=F_{\omega}^{1} \circ \cdots \circ F_{\omega}^{n}(x) .
\end{aligned}
$$

To make our notation complete, we define $\varphi^{0}(x)=x=\gamma^{0}(x)$.

Remark 1. The maps $\varphi^{n}$ and $\gamma^{n}$ are iterated random functions, (see [16] for a review). Assumptions (d) and (e) above ensure that $G^{k}$ and $F^{k}$ are contracting on average. Thus, $\left\{\varphi^{n}\right\}_{n \geq 0}$ and $\left\{\gamma^{n}\right\}_{n \geq 0}$ are Markov chains with invariant probability distributions given by the distributions of the almost sure limits of $\varphi^{-n}$ and $\gamma^{-n}$ as $n \rightarrow \infty$, respectively. Moreover, the distributions of the Markov chains $\varphi^{n}$ and $\gamma^{n}$ converge at a geometric rate to these invariant distributions. These results are immediately attained by applying theorems in, for example, [16, 25, 18]. Nonetheless, we prove the following proposition to make our results more self-contained.

\section{Proposition 1. Define}

$$
Y_{1}(\omega):=\lim _{n \rightarrow \infty} \varphi_{\omega}^{-n}(x) \quad \text { and } \quad Y_{0}(\omega):=\lim _{n \rightarrow \infty} \gamma_{\omega}^{-n}(x) .
$$

These limits exist almost surely and are independent of $x \in X$. 
Remark 2. A random set which attracts all initial data started at " $-\infty$ " and is forward-invariant under the dynamics is called a random pullback attractor [25, 14, 13, 31. When that attractor consists of a single point almost surely then it is called a random point attractor. In this case, the single point can be viewed as a random variable. Random variables such as these are often called random pullback attractors, or "pullbacks" for short, because they take an initial condition $x$ and pull it back to the infinite past [13, 36, 30. Since when the random attractor is a single point one can associate to each realization of random "forcing" a single attracting solution which gives the asymptotic behavior, this is also ofter referred to as the "one force, one solution" paradigm [39, 30, 31.

Proof. We will show that the sequence $\varphi^{-n}(x)$ is almost surely Cauchy. Let $x_{1}, x_{2} \in$ $X$ and $n \geq m$. Using the triangle inequality repeatedly, we obtain

$$
\begin{aligned}
\left|\varphi^{-n}\left(x_{1}\right)-\varphi^{-m}\left(x_{2}\right)\right| & \leq \sum_{i=m+1}^{n}\left|G^{1} \circ \cdots \circ G^{i}\left(x_{1}\right)-G^{1} \circ \cdots \circ G^{i-1}\left(x_{2}\right)\right| \\
& \leq \sum_{i=m+1}^{n}\left|G^{i}\left(x_{1}\right)-x_{2}\right|\left(\prod_{j=1}^{i-1} K_{0}\left(\tau_{0}^{j}\right) K_{1}\left(\tau_{1}^{j}\right)\right) .
\end{aligned}
$$

Observe that

$$
\begin{aligned}
& \mathbb{E} \sum_{i=m+1}^{n}\left|G^{i}\left(x_{1}\right)-x_{2}\right|\left(\prod_{j=1}^{i-1} K_{0}\left(\tau_{0}^{j}\right) K_{1}\left(\tau_{1}^{j}\right)\right) \\
& =\mathbb{E}\left|G^{1}\left(x_{1}\right)-x_{2}\right| \sum_{i=m+1}^{n}\left(\mathbb{E} K_{0}\left(\tau_{0}\right) \mathbb{E} K_{1}\left(\tau_{1}\right)\right)^{i-1} \leq \frac{\mathbb{E}\left|G^{1}\left(x_{1}\right)-x_{2}\right|}{1-\mathbb{E} K_{0}\left(\tau_{0}\right) \mathbb{E} K_{1}\left(\tau_{1}\right)}<\infty,
\end{aligned}
$$

since $\mathbb{E} K_{0}\left(\tau_{0}\right) \mathbb{E} K_{1}\left(\tau_{1}\right)<1$ by assumption, where $\tau_{0}$ and $\tau_{1}$ are independent draws from $\mu_{0}$ and $\mu_{1}$. Thus, $\sum_{i=1}^{\infty}\left|G^{1} \circ \cdots \circ G^{i}\left(x_{1}\right)-G^{1} \circ \cdots \circ G^{i-1}\left(x_{2}\right)\right|$ converges almost surely. Therefore $\varphi^{-n}\left(x_{1}\right)$ is almost surely Cauchy and thus $Y_{1}$ exists almost surely. Since $x_{1}$ and $x_{2}$ were arbitrary, $Y_{1}$ is independent of the $x$ used in its definition. The proof for $Y_{0}$ is similar.

The random variables $Y_{1}$ and $Y_{0}$ satisfy the following invariance properties.

Proposition 2. Let $\tau_{0}$ and $\tau_{1}$ be independent draws from $\mu_{0}$ and $\mu_{1}$. Then

$$
Y_{0}={ }_{d} \Phi_{\tau_{0}}^{0}\left(Y_{1}\right) \quad \text { and } \quad Y_{1}={ }_{d} \Phi_{\tau_{1}}^{1}\left(Y_{0}\right)
$$

where $=_{d}$ denotes equal in distribution.

Proof. Let $y \in X$ and observe that for any $n \in \mathbb{N}$, we have that

$$
\gamma^{-n}(y)={ }_{d} \Phi_{\tau_{0}}^{0}\left(\varphi^{n-1}\left(\Phi_{\tau_{1}}^{1}(y)\right)\right) .
$$

Taking the limit as $n \rightarrow \infty$ yields

$$
\lim _{n \rightarrow \infty} \gamma^{-n}(y)={ }_{d} \lim _{n \rightarrow \infty} \Phi_{\tau_{0}}^{0}\left(\varphi^{n-1}\left(\Phi_{\tau_{1}}^{1}(y)\right)\right)=\Phi_{\tau_{0}}^{0}\left(\lim _{n \rightarrow \infty} \varphi^{n-1}\left(\Phi_{\tau_{1}}^{1}(y)\right)\right)
$$

since $\Phi_{t}^{0}(x)$ is continuous in $x$ for each $t$. Recalling that the definitions of $Y_{0}$ and $Y_{1}$ in Equation (3) are independent of $x$ by Proposition 10 we have that Equation (5) becomes $Y_{0}={ }_{d} \Phi_{\tau_{0}}^{0}\left(Y_{1}\right)$. The proof that $Y_{1}={ }_{d} \Phi_{\tau_{1}}^{1}\left(Y_{0}\right)$ is similar.

Proposition 3. Suppose there exists a nonempty set $S \subset X$ so that for all $t \geq 0$, $\Phi_{t}^{i}: S \rightarrow S$ for $i=0$ and 1. Then $Y_{0}$ and $Y_{1}$ are in the closure, $\bar{S}$, almost surely. 
Proof. If $x \in S$, then $\varphi^{-n}(x) \in S$ almost surely for all $n \geq 0$ by assumption. Thus, $\lim _{n \rightarrow \infty} \varphi^{-n}(x)=Y_{1} \in \bar{S}$ almost surely. But by Proposition 1 , the random variable $Y_{1}$ is independent of the initial $x$ used in its definition, so $Y_{1} \in \bar{S}$ almost surely. The proof for $Y_{0}$ is similar.

2.2. Continuous-time process. To define the continuous time process, we need more notation. Much of the following notation is standard in renewal theory. For each $\omega \in \Omega$ and natural number $n$, define

$$
S_{n}:=\sum_{k=1}^{n} \tau_{0}^{k}+\tau_{1}^{k}
$$

with $S_{0}:=0$. Define $S_{n+1}^{\prime}:=S_{n}+\tau_{0}^{n+1}$ for $n \geq 0$. Observe that $S_{n}^{\prime}<S_{n}<$ $S_{n+1}^{\prime}<S_{n+1}$. Define

$$
N_{t}:=\max \left\{n \geq 0: S_{n} \leq t\right\} .
$$

We also define the state process $J_{t}$ for $t \geq 0$ by

$$
J_{t}:= \begin{cases}0 & S_{N_{t}} \leq t<S_{N_{t}+1}^{\prime} \\ 1 & S_{N_{t}+1}^{\prime} \leq t\end{cases}
$$

Finally, for $t \geq 0$, define the elapsed time since the last switch, often called the age process, by

$$
a_{t}:=J_{t}\left(t-S_{N_{t}+1}^{\prime}\right)+\left(1-J_{t}\right)\left(t-S_{N_{t}}\right) .
$$

We are now ready to define our continuous-time $X$-valued process. For $u_{0} \in X$, $\omega \in \Omega$, and $t \geq 0$, define

$$
u(t, \omega)=J_{t} \Phi_{a_{t}}^{1} \circ \Phi_{\tau_{0}^{N_{t}+1}}^{0}\left(\varphi^{N_{t}}\left(u_{0}\right)\right)+\left(1-J_{t}\right) \Phi_{a_{t}}^{0}\left(\varphi^{N_{t}}\left(u_{0}\right)\right) .
$$

2.3. Convergence in distribution to mixture of pullbacks. In this section we will find the limiting distribution of $u(t)$ as $t \rightarrow \infty$. In order to describe this limiting distribution, we will need to define three more random variables. Define $a^{0}$ and $a^{1}$ to be two random variables with the following cumulative distribution functions:

$$
\mathbb{P}\left(a^{0} \leq x\right)=\frac{\mathbb{E} \min \left(\tau_{0}, x\right)}{\mathbb{E} \tau_{0}} \quad \text { and } \quad \mathbb{P}\left(a^{1} \leq x\right)=\frac{\mathbb{E} \min \left(\tau_{1}, x\right)}{\mathbb{E} \tau_{1}}
$$

We will see in Lemma 1 that the distributions of $a^{0}$ and $a^{1}$ can be thought of as the limiting distributions of the age process conditioned on either $J_{t}=0$ or 1. Let $\xi$ be a Bernoulli random variable with parameter $p:=\left(\mathbb{E} \tau_{1}\right) /\left(\mathbb{E} \tau_{0}+\mathbb{E} \tau_{1}\right)$, the probability that $J_{t}=1$ at large time. Assume $a^{0}, a^{1}$, and $\xi$ are all chosen to be mutually independent and independent of $\left(\tau_{0}^{k}, \tau_{1}^{k}\right)$ for every $k$. Recall that a measure $\mu$ on the real line is said to be arithmetic if there exist a $d>0$ so that $\mu(\{0, d, 2 d, \ldots\})=1$.

Theorem 1. Suppose $\Phi_{t}^{0}$ and $\Phi_{t}^{1}$ satisfy assumptions (a)-(e) of Section [2.1. Let $u(t)$ be defined as in Equation (7), and $a^{0}, a^{1}$, and $\xi$ as in the above paragraph. If the switching time distributions, $\mu_{0}$ and $\mu_{1}$, are non-arithmetic, then we have the following convergence in distribution as $t \rightarrow \infty$.

$$
u(t) \rightarrow_{d} \bar{u}:=\xi \Phi_{a^{1}}^{1}\left(Y_{0}\right)+(1-\xi) \Phi_{a^{0}}^{0}\left(Y_{1}\right) \quad \text { as } t \rightarrow \infty .
$$


If the switching time distributions, $\mu_{0}$ and $\mu_{1}$, are exponential, then we have the following corollary that follows immediately from Proposition 2 and Theorem 1 since the age of a Poisson process is exponentially distributed.

Corollary 1. Suppose the switching time distributions, $\mu_{0}$ and $\mu_{1}$, are exponential with respective rate parameters $r_{0}$ and $r_{1}$. If $\xi$ is Bernoulli with parameter $r_{0} /\left(r_{0}+\right.$ $\left.r_{1}\right)$, then we have the following convergence in distribution as $t \rightarrow \infty$.

$$
u(t) \rightarrow_{d} \bar{u}:=\xi Y_{1}+(1-\xi) Y_{0} \quad \text { as } t \rightarrow \infty .
$$

Proof of Theorem 1] In light of Proposition 2, it is enough to prove that $u(t)$ converges in distribution to $\Phi_{a^{1}}^{1} \circ \Phi_{\tau_{0}}^{0}\left(Y_{1}\right)+(1-\xi) \Phi_{a^{0}}^{0}\left(Y_{1}\right)$, where $\tau_{0}$ is an independent draw from $\mu_{0}$. We will show that for any $A, B$, and $C$ Borel subsets of $\mathbb{R}$ and $D$ a Borel subset of $X$, we have that

$$
\begin{aligned}
\mathbb{P}\left(a_{t} \in A, J_{t} \tau_{0}^{N_{t}+1} \in B, J_{t} \in C, \varphi^{N_{t}}\left(u_{0}\right) \in D\right) \\
\quad \rightarrow \mathbb{P}\left(\xi a^{1}+(1-\xi) a^{0} \in A, \xi \tau_{0} \in B, \xi \in C, Y_{1} \in D\right) \quad \text { as } \quad t \rightarrow \infty .
\end{aligned}
$$

Once this convergence is shown, the conclusion of the theorem quickly follows. To see this, assume the convergence in Equation (8) holds. Define the $\left(\mathbb{R}^{3} \times X\right)$ valued random variable $Y_{t}:=\left(a_{t}, J_{t} \tau_{0}^{N_{t}+1}, J_{t}, \varphi^{N_{t}}\left(u_{0}\right)\right)$. Since $X$ is assumed to be separable, the product $\mathbb{R}^{3} \times X$ is separable and thus we can apply Theorem 2.8 in [7] to obtain that $Y_{t}$ converges in distribution to $\left(\xi a^{1}+(1-\xi) a^{0}, \xi \tau_{0}, \xi, Y_{1}\right)$ as $t \rightarrow \infty$. Define the function $g: \mathbb{R}^{3} \times X \rightarrow X$ by $g(a, t, j, y)=j \Phi_{a}^{1} \circ \Phi_{t}^{0}(y)+(1-j) \Phi_{t}^{0}(y)$ and observe that $u(t)=g\left(a_{t}, \tau_{0}^{N_{t+1}}, J_{t}, \varphi^{N_{t}}\right)$ and $\bar{u}=g\left(a^{1}, \tau_{0}, \xi, Y\right)$. Since $g$ is continuous, the conclusion of the theorem follows from the continuous mapping theorem (see, for example, Theorem 3.2.4 in [19]). Therefore, it remains only to show the convergence in Equation (8).

In what follows, we will make extensive use of indicator functions. For ease of reading, we will often denote the indicator $1_{A}=1_{A}(\omega)$ by $\{A\}=\{A\}(\omega)$.

For each $t \geq 0$, define $\mathcal{F}_{t}$ to be the $\sigma$-algebra generated by $S_{N_{t}}$ and $\left\{\left(\tau_{0}^{k}, \tau_{1}^{k}\right)\right\}_{k=N_{t}+1}^{\infty}$. Since $a_{t}, \tau_{0}^{N_{t}+1}$, and $J_{t}$ are measurable with respect to $\mathcal{F}_{t}$, the tower property of conditional expectation and the triangle inequality give

$$
\begin{aligned}
& \mid \mathbb{E}\left\{a_{t} \in A, J_{t} \tau_{0}^{N_{t}+1} \in B, J_{t} \in C, \varphi^{N_{t}} \in D\right\} \\
& -\mathbb{E}\left\{\xi a^{1}+(1-\xi) a^{0} \in A, \xi \tau_{0} \in B, \xi \in C, Y_{1} \in D\right\} \mid \\
& \leq \mid \mathbb{E}\left[\left\{a_{t} \in A, J_{t} \tau_{0}^{N_{t}+1} \in B, J_{t} \in C\right\} \mathbb{E}\left[\left\{\varphi^{N_{t}} \in D\right\} \mid \mathcal{F}_{t}\right]\right] \\
& -\mathbb{E}\left[\left\{a_{t} \in A, J_{t} \tau_{0}^{N_{t}+1} \in B, J_{t} \in C\right\} \mathbb{E}\left[\left\{Y_{1} \in D\right\}\right]\right] \mid \\
& +\mid \mathbb{E}\left[\left\{a_{t} \in A, J_{t} \tau_{0}^{N_{t}+1} \in B, J_{t} \in C\right\} \mathbb{E}\left[\left\{Y_{1} \in D\right\}\right]\right] \\
& \quad-\mathbb{E}\left\{\xi a^{1}+(1-\xi) a^{0} \in A, \xi \tau_{0} \in B, \xi \in C\right\}\left\{Y_{1} \in D\right\} \mid .
\end{aligned}
$$

By Lemma 6, we have that $\mathbb{E}\left[\left\{\varphi^{N_{t}} \in D\right\} \mid \mathcal{F}_{t}\right] \rightarrow \mathbb{E}\left[\left\{Y_{1} \in D\right\}\right]$ almost surely as $t \rightarrow \infty$. Therefore, the first term goes to 0 by the dominated convergence theorem. Since $Y_{1}$ is independent of $\xi, a^{1}, a^{0}$, and $\tau_{0}$, the second term is bounded above by

$$
\left|\mathbb{E}\left\{a_{t} \in A, J_{t} \tau_{0}^{N_{t}+1} \in B, J_{t} \in C\right\}-\mathbb{E}\left\{\xi a^{1}+(1-\xi) a^{0} \in A, \xi \tau_{0} \in B, \xi \in C\right\}\right|
$$


To show that (9) goes to 0 as $t \rightarrow \infty$, we consider the four possible cases for the inclusion of 0 and 1 in $C$. If both 0 and 1 are not in $C$, then (9) is 0 for all $t \geq 0$ since $J_{t}$ and $\xi$ are each almost surely 0 or 1 .

Suppose $0 \in C$ and $1 \notin C$. Then the indicator function in the first term of (9) is only non-zero if $J_{t}=0$. Hence, we can replace $\left\{J_{t} \in C\right\}$ by $\left(1-J_{t}\right)$ and $\left\{J_{t} \tau_{0}^{N_{t}+1} \in B\right\}$ by $\{0 \in B\}$. Similarly, in the second term we replace $\{\xi \in C\}$ by $(1-\xi),\left\{\xi \tau_{0} \in B\right\}$ by $\{0 \in B\}$, and $\left\{\xi a^{1}+(1-\xi) a^{0} \in A\right\}$ by $\left\{a^{0} \in A\right\}$. Thus (9) becomes

$$
\begin{aligned}
\text { (9) } & =\left|\mathbb{E}\left\{a_{t} \in A, 0 \in B\right\}\left(1-J_{t}\right)-\mathbb{E}\left\{a^{0} \in A, 0 \in B\right\}(1-\xi)\right| \\
& \leq\left|\mathbb{E}\left\{a_{t} \in A\right\}\left(1-J_{t}\right)-\mathbb{E}\left\{a^{0} \in A\right\}(1-\xi)\right| .
\end{aligned}
$$

By Lemma 1, this term goes to 0 as $t \rightarrow \infty$.

Suppose $1 \in C$ and $0 \notin C$. Then the indicator function in the first term of (9) is only non-zero if $J_{t}=1$. Thus after performing similar replacements to those above, (9) becomes

$$
\text { (9) }=\left|\mathbb{E}\left\{a_{t} \in A, \tau_{0}^{N_{t}+1} \in B\right\} J_{t}-\mathbb{E}\left\{a^{1} \in A, \tau_{0} \in B\right\} \xi\right| \text {. }
$$

Define $\mathcal{F}_{t}^{\prime}$ to be the $\sigma$-algebra generated by $S_{N_{t}+1}^{\prime}, \tau_{1}^{N_{t}+1}$, and $\left\{\left(\tau_{0}^{k}, \tau_{1}^{k}\right)\right\}_{k=N_{t}+2}^{\infty}$. Observe that $J_{t}$ and $a_{t}$ are both measurable with respect to $\mathcal{F}_{t}^{\prime}$. Therefore, by the tower property of conditional expectation and the triangle inequality we have that

$$
\begin{aligned}
&\left|\mathbb{E}\left\{a_{t} \in A, \tau_{0}^{N_{t}+1} \in B\right\} J_{t}-\mathbb{E}\left\{a^{1} \in A, \tau_{0} \in B\right\} \xi\right| \\
& \leq\left|\mathbb{E}\left[\left\{a_{t} \in A\right\} J_{t} \mathbb{E}\left[\left\{\tau_{0}^{N_{t}+1} \in B\right\} \mid \mathcal{F}_{t}^{\prime}\right]\right]-\mathbb{E}\left[\left\{a_{t} \in A\right\} J_{t} \mathbb{E}\left[\left\{\tau_{0} \in B\right\}\right]\right]\right| \\
&+\left|\mathbb{E}\left[\left\{a_{t} \in A\right\} J_{t} \mathbb{E}\left[\left\{\tau_{0} \in B\right\}\right]\right]-\mathbb{E}\left\{a^{1} \in A, \tau_{0} \in B\right\} \xi\right| .
\end{aligned}
$$

Lemma 7 gives us that $J_{t} \mathbb{E}\left[\left\{\tau_{0}^{N_{t}+1} \in B\right\} \mid \mathcal{F}^{\prime}{ }_{t}\right]=J_{t} \mathbb{E}\left[\left\{\tau_{0}^{1} \in B\right\} \mid \mathcal{F}^{\prime}{ }_{t}\right]$ almost surely and Lemma 8 gives that $\mathbb{E}\left[\left\{\tau_{0}^{1} \in B\right\} \mid \mathcal{F}^{\prime}{ }_{t}\right] \rightarrow \mathbb{E}\left[\left\{\tau_{0} \in B\right\}\right]$ almost surely as $t \rightarrow \infty$. Therefore, the first term goes to 0 as $t \rightarrow \infty$ by the dominated convergence theorem. Finally since $\tau_{0}$ is independent of $\xi$ and $a^{1}$, we have the following bound on the second term

$$
\left|\mathbb{E}\left[\left\{a_{t} \in A\right\} J_{t} \mathbb{E}\left[\left\{\tau_{0} \in B\right\}\right]\right]-\mathbb{E}\left\{a^{1} \in A, \tau_{0} \in B\right\} \xi\right| \leq\left|\mathbb{E}\left\{a_{t} \in A\right\} J_{t}-\mathbb{E}\left\{a^{1} \in A\right\} \xi\right|
$$

This goes to 0 as $t \rightarrow \infty$ by Lemma 1 .

Finally, if both $0 \in C$ and $1 \in C$, then (9) becomes

$$
\begin{aligned}
& \text { (9) }=\left|\mathbb{E}\left\{a_{t} \in A, J_{t} \tau_{0}^{N_{t}+1} \in B\right\}-\mathbb{E}\left\{\xi a^{1}+(1-\xi) a^{0} \in A, \xi \tau_{0} \in B\right\}\right| \\
& \leq\left|\mathbb{E}\left\{a_{t} \in A, \tau_{0}^{N_{t}+1} \in B\right\} J_{t}-\mathbb{E}\left\{a^{1} \in A, \tau_{0} \in B\right\} \xi\right| \\
& +\left|\mathbb{E}\left\{a_{t} \in A, 0 \in B\right\}\left(1-J_{t}\right)-\mathbb{E}\left\{a^{0} \in A, 0 \in B\right\}(1-\xi)\right| .
\end{aligned}
$$

We've already shown that each of these terms go to zero as $t \rightarrow \infty$, so the proof is complete.

2.4. The Lemmas. We now state and prove all of the lemmas that are needed for Theorem 1. This first lemma calculates the limiting distribution of the age process. It can be interpreted as first flipping a coin to determine if $J_{t}$ is 0 or 1 , and then choosing from the limiting distribution of the age conditioned on $J_{t}$. 
Lemma 1. We have that $a_{t} \rightarrow_{d} \xi a^{1}+(1-\xi) a^{0}$ as $t \rightarrow \infty$. In particular, for any $x \geq 0$ with $A:=(-\infty, x]$, we have that as $t \rightarrow \infty$

$$
\left|\mathbb{E}\left\{a_{t} \in A\right\} J_{t}-\mathbb{E}\left\{a^{1} \in A\right\} \xi\right|+\left|\mathbb{E}\left\{a_{t} \in A\right\}\left(1-J_{t}\right)-\mathbb{E}\left\{a^{0} \in A\right\}(1-\xi)\right| \rightarrow 0 .
$$

Proof. Let $x \geq 0$ and define $A:=(-\infty, x]$. Observe the following bound

$$
\begin{aligned}
& \left|\mathbb{E}\left\{a_{t} \in A\right\}-\mathbb{E}\left\{\xi a^{1}+(1-\xi) a^{0} \in A\right\}\right| \\
& \quad \leq\left|\mathbb{E}\left\{a_{t} \in A\right\} J_{t}-\mathbb{E}\left\{a^{1} \in A\right\} \xi\right|+\left|\mathbb{E}\left\{a_{t} \in A\right\}\left(1-J_{t}\right)-\mathbb{E}\left\{a^{0} \in A\right\}(1-\xi)\right| .
\end{aligned}
$$

We will show that the first term goes to zero. The second term goes to zero by an analogous argument.

For our given $x \geq 0$, consider the alternating renewal process that is said to be "on" when $0 \leq t-S_{N_{t}+1}^{\prime} \leq x$ and "off" otherwise. Formally, we define the "on/off" state process

$$
b_{t}= \begin{cases}1 & \text { if } 0 \leq t-S_{N_{t}+1}^{\prime} \leq x \\ 0 & \text { otherwise }\end{cases}
$$

Observe that the lengths of time that the process is "on" are $\left\{\min \left(\tau_{1}^{k}, x\right)\right\}_{k=1}^{\infty}$. Similarly, the lengths of time that the process is "off" are $\tau_{0}^{1}$ and $\left\{\tau_{0}^{k}+\left(\tau_{1}^{k-1}-x\right)^{+}\right\}_{k=2}^{\infty}$, where as usual $(y)^{+}$is equal to $y$ if $y \geq 0$ and 0 otherwise. Since the distribution of $\min \left(\tau_{1}^{k}, x\right)+\tau_{0}^{k}+\left(\tau_{1}^{k-1}-x\right)^{+}$is nonarithmetic, and since $\mathbb{E}\left[\min \left(\tau_{1}^{k}, x\right)+\tau_{0}^{k}+\right.$ $\left.\left(\tau_{1}^{k-1}-x\right)^{+}\right]<\infty$, we can apply Theorem 3.4.4 in [35] to obtain

$$
\lim _{t \rightarrow \infty} \mathbb{P}\left(b_{t}=1\right)=\frac{\mathbb{E} \min \left(\tau_{1}, x\right)}{\mathbb{E}\left[\min \left(\tau_{1}^{k}, x\right)+\tau_{0}^{k}+\left(\tau_{1}^{k-1}-x\right)^{+}\right]} .
$$

Informally, this intuitive result states that the probability that the alternating renewal process is "on" at large time is just the expected length of an "on" bout divided by the sum of the expected lengths of an "off" bout and an "on" bout. Since $\mathbb{E}\left[\min \left(\tau_{1}^{k}, x\right)+\tau_{0}^{k}+\left(\tau_{1}^{k-1}-x\right)^{+}\right]=\mathbb{E} \tau_{0}+\mathbb{E} \tau_{1}$ and since the distribution of $a^{1}$ is chosen so that $\mathbb{E} \tau_{1} \mathbb{P}\left(a^{1} \leq x\right)=\mathbb{E} \min \left(\tau_{1}, x\right)$, Equation (10) simplifies to

$$
\lim _{t \rightarrow \infty} \mathbb{P}\left(b_{t}=1\right)=\frac{\mathbb{E} \tau_{1} \mathbb{P}\left(a^{1} \leq x\right)}{\mathbb{E} \tau_{0}+\mathbb{E} \tau_{1}} .
$$

Therefore

$$
\begin{aligned}
\mathbb{E}\left[\left\{a_{t} \leq x\right\} J_{t}\right] & =\mathbb{P}\left(a_{t} \leq x, J_{t}=1\right)=\mathbb{P}\left(0 \leq t-S_{N_{t}+1} \leq x\right) \\
& =\mathbb{P}\left(b_{t}=1\right) \underset{t \rightarrow \infty}{\longrightarrow} \frac{\mathbb{E} \tau_{1} \mathbb{P}\left(a^{1} \leq x\right)}{\mathbb{E} \tau_{0}+\mathbb{E} \tau_{1}}=\mathbb{E}\left[\left\{a^{1} \leq x\right\} \xi\right] .
\end{aligned}
$$

The last equality holds because $\xi$ and $a^{1}$ are independent and $\mathbb{E} \xi=\mathbb{E} \tau_{1} /\left(\mathbb{E} \tau_{0}+\mathbb{E} \tau_{1}\right)$.

The analogous argument shows that $\left|\mathbb{E}\left\{a_{t} \in A\right\}\left(1-J_{t}\right)-\mathbb{E}\left\{a^{0} \in A\right\}(1-\xi)\right| \rightarrow 0$ as $t \rightarrow \infty$ and the proof is complete.

The next three lemmas are general results that are all relatively standard. We return to lemmas specific to our problem in Lemma 5 .

Lemma 2. Suppose $X_{t} \rightarrow X_{\infty}$ a.s. as $t \rightarrow \infty$ and $X_{t} \leq B$ a.s. where $B$ is a random variable satisfying $\mathbb{E} B<\infty$. If $\mathcal{F}_{t} \subset \mathcal{F}_{s}$ for $0 \leq s \leq t$, and $\mathcal{F}_{\infty}:=\cap_{t \geq 0} \mathcal{F}_{t}$, then

$$
\mathbb{E}\left[X_{t} \mid \mathcal{F}_{t}\right] \rightarrow \mathbb{E}\left[X_{\infty} \mid \mathcal{F}_{\infty}\right] \quad \text { almost surely as } t \rightarrow \infty
$$


Proof. We first show the convergence for an $X=X_{t}$ independent of $t$. Let $X$ be any integrable random variable and for $t \leq 0$ define

$$
M_{t}:=\mathbb{E}\left[X \mid \mathcal{F}_{-t}\right] \text {. }
$$

We claim that $\left\{M_{t}\right\}_{t=0}^{-\infty}$ is a backwards martingale. For $s \leq t \leq 0$ we have that $\mathcal{F}_{-s} \subset \mathcal{F}_{-t}$ and therefore by the tower property of conditional expectation,

$$
\mathbb{E}\left[M_{t} \mid \mathcal{F}_{-s}\right]=\mathbb{E}\left[\mathbb{E}\left[X \mid \mathcal{F}_{-t}\right] \mid \mathcal{F}_{-s}\right]=\mathbb{E}\left[X \mid \mathcal{F}_{-s}\right]=M_{s} .
$$

Since by definition of conditional expectation $M_{t} \in \mathcal{F}_{-t}$, and since $M_{t} \leq B$ almost surely where $\mathbb{E} B<\infty$, we have that $M_{t}$ is indeed a backwards martingale. By the backwards martingale convergence theorem, $M_{-\infty}:=\lim _{t \rightarrow-\infty} M_{t}$ exists almost surely and in $L^{1}(\Omega)$.

We claim that $M_{-\infty}=\mathbb{E}\left[X \mid \mathcal{F}_{\infty}\right]$. Since for $t \leq T \leq 0$ we have that $M_{t} \in \mathcal{F}_{-t} \subset$ $\mathcal{F}_{-T}$, it follows that $M_{-\infty} \in \mathcal{F}_{-T}$. Since $T \leq 0$ was arbitrary, $M_{-\infty} \in \mathcal{F}_{\infty}$.

Let $A \in \mathcal{F}_{\infty}$. Then

$\left|\mathbb{E} M_{-t} 1_{A}-\mathbb{E} M_{-\infty} 1_{A}\right| \leq \mathbb{E}\left|M_{-t} 1_{A}-M_{-\infty} 1_{A}\right| \leq \mathbb{E}\left|M_{-t}-M_{-\infty}\right| \rightarrow 0 \quad$ as $t \rightarrow \infty$ since $M_{-t} \rightarrow M_{-\infty}$ in $L^{1}(\Omega)$. But,

$$
\mathbb{E} M_{-t} 1_{A}=\mathbb{E}\left[\mathbb{E}\left[X \mid \mathcal{F}_{t}\right] 1_{A}\right]=\mathbb{E}\left[\mathbb{E}\left[X 1_{A} \mid \mathcal{F}_{t}\right]\right]=\mathbb{E} X 1_{A} .
$$

Therefore $\mathbb{E} X 1_{A}=\mathbb{E} M_{-\infty} 1_{A}$, and so we conclude that $M_{-\infty}=\mathbb{E}\left[X \mid \mathcal{F}_{\infty}\right]$.

We now show the convergence for the case where $X_{t}$ depends on $t$. Let $T \geq 0$ and define $B_{T}:=\sup \left\{\left|X_{t}-X_{s}\right|: t, s>T\right\} . B_{T} \leq 2 B$, so $B_{T}$ is integrable. Thus,

$$
\limsup _{t \rightarrow \infty} \mathbb{E}\left[\left|X_{t}-X_{\infty}\right| \mathcal{F}_{t}\right] \leq \lim _{t \rightarrow \infty} \mathbb{E}\left[B_{T} \mid \mathcal{F}_{t}\right]=\mathbb{E}\left[B_{T} \mid \mathcal{F}_{\infty}\right]
$$

By assumption, $B_{T} \rightarrow 0$ a.s. as $T \rightarrow \infty$ so by Jensen's inequality

$$
\left|\mathbb{E}\left[X_{t} \mid \mathcal{F}_{t}\right]-\mathbb{E}\left[X_{\infty} \mid \mathcal{F}_{t}\right]\right| \leq \mathbb{E}\left[\mid X_{t}-X_{\infty} \| \mathcal{F}_{t}\right] \rightarrow 0 .
$$

Therefore,

$$
\left|\mathbb{E}\left[X_{t} \mid \mathcal{F}_{t}\right]-\mathbb{E}\left[X_{\infty} \mid \mathcal{F}_{\infty}\right]\right| \leq\left|\mathbb{E}\left[X_{t} \mid \mathcal{F}_{t}\right]-\mathbb{E}\left[X_{\infty} \mid \mathcal{F}_{t}\right]\right|+\left|\mathbb{E}\left[X_{\infty} \mid \mathcal{F}_{t}\right]-\mathbb{E}\left[X_{\infty} \mid \mathcal{F}_{\infty}\right]\right| .
$$

We've just shown that the first term goes to 0 , and we've shown that the second term goes to 0 since $X_{\infty}$ doesn't depend on $t$, so the proof is complete.

Lemma 3. If $X_{n} \rightarrow X_{\infty}$ a.s. as $n \rightarrow \infty$ and $N_{t} \rightarrow \infty$ a.s. as $t \rightarrow \infty$, then

$$
X_{N_{t}} \rightarrow X_{\infty} \quad \text { a.s. as } t \rightarrow \infty \text {. }
$$

Proof. Let $A:=\left\{X_{n} \nrightarrow X_{\infty}\right\}$ and $B:=\left\{N_{t} \nrightarrow \infty\right\}$. Then

$$
\mathbb{P}\left(X_{N_{t}} \nrightarrow X_{\infty}\right) \leq \mathbb{P}(A \cup B) \leq \mathbb{P}(A)+\mathbb{P}(B)=0 .
$$

We now give some standard definitions. Let $(\Omega, \mathcal{F}, P)$ be a probability space. A measurable map $\pi: \Omega \rightarrow \Omega$ is said to be measure preserving if $\mathbb{P}\left(\pi^{-1} A\right)=\mathbb{P}(A)$ for all $A \in \mathcal{F}$. Let $\pi$ be a given measure preserving map. A set $A \in \mathcal{F}$ is said to be $\pi$-invariant if $\pi^{-1} A=A$, where two sets are considered to be equal if their symmetric difference has probability 0 . A random variable $X$ is said to be $\pi$-invariant if $X=X \circ \pi$ almost surely.

Lemma 4. Let $\pi: \Omega \rightarrow \Omega$ be a measure preserving map. If $X$ is $\pi$-invariant, then so is every set in its $\sigma$-algebra.

Proof. See, for example, [19] Exercise 7.1.1. 
Lemma 5. For each $t \geq 0$ define $\mathcal{F}_{t}$ to be the $\sigma$-algebra generated by $S_{N_{t}}$ and $\left\{\left(\tau_{0}^{k}, \tau_{1}^{k}\right)\right\}_{k=N_{t}+1}^{\infty}$. If $D$ is a Borel set of $X$, then for each $t \geq 0$

$$
\mathbb{E}\left[\left\{\varphi^{N_{t}} \in D\right\} \mid \mathcal{F}_{t}\right]=\mathbb{E}\left[\left\{\varphi^{-N_{t}} \in D\right\} \mid \mathcal{F}_{t}\right] \quad \text { a.s. }
$$

Remark 3. To see why this lemma should be true, observe that (a) the random variables $\varphi^{N_{t}}$ and $\varphi^{-N_{t}}$ are equal after a re-ordering of the first $N_{t}$-many $\omega_{k}$ 's and that (b) the random variables generating $\mathcal{F}_{t}$ don't depend on the order of the first $N_{t}$-many $\omega_{k}$ 's.

Proof. Fix a $t \geq 0$ and let $A \in \mathcal{F}_{t}$. By the definition of conditional expectation, we have that

$$
\int_{\Omega} \mathbb{E}\left[\left\{\varphi^{N_{t}} \in D\right\} \mid \mathcal{F}_{t}\right](\omega)\{A\}(\omega) d \mathbb{P}=\int_{\Omega}\left\{\varphi^{N_{t}} \in D\right\}(\omega)\{A\}(\omega) d \mathbb{P} .
$$

Define $\sigma_{t}: \Omega \rightarrow \Omega$ to be the permutation that inverts the order of the first $N_{t^{-}}$ many $\omega_{k}$ 's. That is, $\left(\sigma_{t}(\omega)\right)_{k}=\omega_{N_{t}-k+1}$ for $k \in\left\{1, \ldots, N_{t}\right\}$ and $\left(\sigma_{t}(\omega)\right)_{k}=\omega_{k}$ for $k>N_{t}$. Observe that $N_{t}(\omega)=N_{t}\left(\sigma_{t}(\omega)\right)$ and thus $\varphi^{N_{t}}(\omega)=\varphi^{-N_{t}}\left(\sigma_{t}(\omega)\right)$. Also, $S_{N_{t}}$ and $\left\{\left(\tau_{0}^{k}, \tau_{1}^{k}\right)\right\}_{k=N_{t}+1}^{\infty}$ are $\sigma_{t}$-invariant, so $A$ is $\sigma_{t}$-invariant by Lemma 4. Thus

$$
\int_{\Omega}\left\{\varphi^{N_{t}} \in D\right\}(\omega)\{A\}(\omega) d \mathbb{P}=\int_{\Omega}\left\{\varphi^{-N_{t}} \in D\right\}\left(\sigma_{t}(\omega)\right)\{A\}\left(\sigma_{t}(\omega)\right) d \mathbb{P} .
$$

Since $\sigma_{t}$ is measure preserving and by the definition of conditional expectation,

$$
\begin{aligned}
\int_{\Omega}\left\{\varphi^{-N_{t}} \in D\right\}\left(\sigma_{t}(\omega)\right)\{A\}\left(\sigma_{t}(\omega)\right) d \mathbb{P} & =\int_{\Omega}\left\{\varphi^{-N_{t}} \in D\right\}(\omega)\{A\}(\omega) d \mathbb{P} \\
& =\int_{\Omega} \mathbb{E}\left[\left\{\varphi^{-N_{t}} \in D\right\} \mid \mathcal{F}_{t}\right](\omega)\{A\}(\omega) d \mathbb{P} .
\end{aligned}
$$

Putting this all together,

$$
\int_{\Omega} \mathbb{E}\left[\left\{\varphi^{N_{t}} \in D\right\} \mid \mathcal{F}_{t}\right]\{A\} d \mathbb{P}=\int_{\Omega} \mathbb{E}\left[\left\{\varphi^{-N_{t}} \in D\right\} \mid \mathcal{F}_{t}\right]\{A\} d \mathbb{P} .
$$

Since $A$ was an arbitrary element of $\mathcal{F}_{t}$, the proof is complete.

Recall that the random variable $Y_{1}$ is defined by $Y_{1}:=\lim _{n \rightarrow \infty} \varphi^{-n}(x)$, and is independent of the choice of $x \in X$, by Proposition 1

Lemma 6. For each $t \geq 0$ define $\mathcal{F}_{t}$ to be the $\sigma$-algebra generated by $S_{N_{t}}$ and $\left\{\left(\tau_{0}^{k}, \tau_{1}^{k}\right)\right\}_{k=N_{t}+1}^{\infty}$. If $D$ is a Borel set of $X$, then with probability one

$$
\begin{aligned}
\mathbb{E}\left[\left\{\varphi^{-N_{t}} \in D\right\} \mid \mathcal{F}_{t}\right] & \rightarrow \mathbb{E}\left\{Y_{1} \in D\right\} \quad \text { as } t \rightarrow \infty \\
\text { and } \quad \mathbb{E}\left[\left\{\varphi^{N_{t}} \in D\right\} \mid \mathcal{F}_{t}\right] & \rightarrow \mathbb{E}\left\{Y_{1} \in D\right\} \quad \text { as } t \rightarrow \infty
\end{aligned}
$$

Proof. In light of Lemma 5, it suffices to show the convergence in Equation (11).

Since $\varphi^{-n} \rightarrow Y_{1}$ almost surely as $n \rightarrow \infty$ and since $N_{t} \rightarrow \infty$ almost surely as $t \rightarrow \infty$, we have that $\varphi^{-N_{t}} \rightarrow Y_{1}$ almost surely by Lemma 3. Define $\mathcal{F}_{\infty}:=\cap_{t \geq 0} \mathcal{F}_{t}$ and observe that $\mathcal{F}_{t} \subset \mathcal{F}_{s}$ for $t \geq s \geq 0$. Thus, by Lemma 2,

$$
\mathbb{E}\left[\left\{\varphi^{-N_{t}} \in D\right\} \mid \mathcal{F}_{t}\right] \rightarrow \mathbb{E}\left[\left\{Y_{1} \in D\right\} \mid \mathcal{F}_{\infty}\right] \quad \text { almost surely as } t \rightarrow \infty .
$$

To complete the proof, we will show that for every $A \in \mathcal{F}_{\infty}, \mathbb{P}(A)=0$ or 1 . To show this, we will show that $\mathcal{F}_{\infty}$ is contained in the exchangeable $\sigma$-algebra and 
then apply the Hewitt-Savage zero-one law. Let $n \in \mathbb{N}, A \in \mathcal{F}_{\infty}$, and $\pi_{n}$ be an arbitrary permutation of $\omega_{1}, \ldots, \omega_{n}$. Define $\pi_{t}: \Omega \rightarrow \Omega$ by

$$
\pi_{t}(\omega)= \begin{cases}\pi_{n}(\omega) & N_{t} \geq n \\ \omega & N_{t}<n .\end{cases}
$$

Since $S_{N_{t}}$ and $\left\{\left(\tau_{0}^{k}, \tau_{1}^{k}\right)\right\}_{k=N_{t}+1}^{\infty}$ are $\pi_{t}$-invariant, then $A$ is $\pi_{t}$-invariant by Lemma 4 as $A \in \mathcal{F}_{\infty} \subset \mathcal{F}_{t}$. Therefore

$$
\mathbb{P}\left(A \Delta \pi_{n}^{-1} A, N_{t} \geq n\right)=\mathbb{P}\left(A \Delta \pi_{t}^{-1} A, N_{t} \geq n\right) \leq \mathbb{P}\left(A \Delta \pi_{t}^{-1} A\right)=0 .
$$

Hence

$$
\begin{aligned}
\mathbb{P}\left(A \Delta \pi_{n}^{-1} A\right) & =\mathbb{P}\left(A \Delta \pi_{n}^{-1} A, N_{t} \geq n\right)+\mathbb{P}\left(A \Delta \pi_{n}^{-1} A, N_{t}<n\right) \\
& \leq \mathbb{P}\left(A \Delta \pi_{n}^{-1} A, N_{t}<n\right) \leq \mathbb{P}\left(N_{t}<n\right) .
\end{aligned}
$$

Since $t$ was arbitrary, and because $\mathbb{P}\left(N_{t}<n\right) \rightarrow 0$ as $t \rightarrow \infty$ since $N_{t} \rightarrow \infty$ almost surely, we conclude that $\mathbb{P}\left(A \Delta \pi_{n}^{-1} A\right)=0$. Since $\pi_{n}$ was an arbitrary finite permutation, we conclude that $\mathcal{F}_{\infty}$ is contained in the exchangeable $\sigma$-algebra. By the Hewitt-Savage zero-one law, $\mathcal{F}_{\infty}$ only contains events that have probability 0 or 1. Thus, $\left\{Y_{1} \in D\right\}$ is trivially independent of $\mathcal{F}_{\infty}$ and therefore $\mathbb{E}\left[\left\{Y_{1} \in D\right\} \mid \mathcal{F}_{\infty}\right]=$ $\mathbb{E}\left\{Y_{1} \in D\right\}$.

Lemma 7. For each $t \geq 0$, define $\mathcal{F}_{t}^{\prime}$ to be the $\sigma$-algebra generated by $S_{N_{t}+1}^{\prime}$, $\tau_{1}^{N_{t}+1}$, and $\left\{\left(\tau_{0}^{k}, \tau_{1}^{k}\right)\right\}_{k=N_{t}+2}^{\infty}$. Then

$$
J_{t} \mathbb{E}\left[\left\{\tau_{0}^{N_{t}+1} \in B\right\} \mid \mathcal{F}^{\prime}{ }_{t}\right]=J_{t} \mathbb{E}\left[\left\{\tau_{0}^{1} \in B\right\} \mid \mathcal{F}^{\prime}{ }_{t}\right] \quad \text { almost surely. }
$$

Remark 4. Recall that $J_{t}$ is either 0 if $S_{N_{t}} \leq t<S_{N_{t}+1}^{\prime}$ or 1 if $S_{N_{t}+1}^{\prime} \leq t$. Hence, this Lemma states that $\mathbb{E}\left[\left\{\tau_{0}^{N_{t}+1} \in B\right\} \mid \mathcal{F}^{\prime}{ }_{t}\right]=\mathbb{E}\left[\left\{\tau_{0}^{1} \in B\right\} \mid \mathcal{F}^{\prime}{ }_{t}\right]$ if $J_{t}=1$.

Remark 5. The proof of this Lemma is very similar to the proof of Lemma 5 ,

Proof. If $\omega$ is such that $J_{t}=0$, then the equality is trivially satisfied. Let $A \in \mathcal{F}_{t}^{\prime}$. Since $\left\{\omega \in \Omega: J_{t}(\omega)=1\right\} \in \mathcal{F}_{t}^{\prime}$, we have by the definition of conditional expectation that

$$
\int_{\Omega} \mathbb{E}\left[\left\{\tau_{0}^{N_{t}+1} \in B\right\} \mid \mathcal{F}_{t}^{\prime}\right]\left\{A, J_{t}=1\right\} d \mathbb{P}=\int_{\Omega}\left\{\tau_{0}^{N_{t}+1} \in B\right\}(\omega)\left\{A, J_{t}=1\right\}(\omega) d \mathbb{P} .
$$

Define $\sigma_{t}: \Omega \rightarrow \Omega$ by

$$
\left(\sigma_{t}(\omega)\right)_{k}= \begin{cases}\left(\tau_{0}^{N_{t}+1}, \tau_{1}^{1}\right) & \text { if } k=1 \text { and } J_{t}=1 \\ \left(\tau_{0}^{1}, \tau_{1}^{N_{t}+1}\right) & \text { if } k=N_{t}+1 \text { and } J_{t}=1 \\ \omega_{k} & \text { otherwise. }\end{cases}
$$

That is, $\sigma_{t}$ switches $\tau_{0}^{1}$ and $\tau_{0}^{N_{t}+1}$ if $J_{t}=1$ and otherwise does nothing. Since $S_{N_{t}+1}^{\prime}$, $\tau_{1}^{N_{t}+1}$, and $\left\{\left(\tau_{0}^{k}, \tau_{1}^{k}\right)\right\}_{k=N_{t}+2}^{\infty}$ are all $\sigma_{t}$-invariant, we have that $A$ is $\sigma_{t}$-invariant by Lemma 4. Also observe that $\left\{J_{t}=1\right\}$ is $\sigma_{t}$-invariant. Thus

$$
\int_{\Omega}\left\{\tau_{0}^{N_{t}+1} \in B\right\}(\omega)\left\{A, J_{t}=1\right\}(\omega) d \mathbb{P}=\int_{\Omega}\left\{\tau_{0}^{1} \in B\right\}\left(\sigma_{t}(\omega)\right)\left\{A, J_{t}=1\right\}\left(\sigma_{t}(\omega)\right) d \mathbb{P} .
$$


Since $\sigma_{t}$ is measure preserving, and by the definition of conditional expectation, we have that

$$
\begin{aligned}
\int_{\Omega}\left\{\tau_{0}^{1} \in B\right\}\left(\sigma_{t}(\omega)\right)\left\{A, J_{t}=1\right\}\left(\sigma_{t}(\omega)\right) d \mathbb{P} & =\int_{\Omega}\left\{\tau_{0}^{1} \in B\right\}(\omega)\left\{A, J_{t}=1\right\}(\omega) d \mathbb{P} \\
& =\int_{\Omega} \mathbb{E}\left[\left\{\tau_{0}^{1} \in B\right\} \mid \mathcal{F}_{t}^{\prime}\right]\left\{A, J_{t}=1\right\} d \mathbb{P} .
\end{aligned}
$$

Putting all this together,

$$
\int_{\Omega} \mathbb{E}\left[\left\{\tau_{0}^{N_{t}+1} \in B\right\} \mid \mathcal{F}_{t}^{\prime}\right]\left\{A, J_{t}=1\right\} d \mathbb{P}=\int_{\Omega} \mathbb{E}\left[\left\{\tau_{0}^{1} \in B\right\} \mid \mathcal{F}_{t}^{\prime}\right]\left\{A, J_{t}=1\right\} d \mathbb{P} .
$$

This implies that $\mathbb{E}\left[\left\{\tau_{0}^{N_{t}+1} \in B\right\} \mid \mathcal{F}^{\prime}{ }_{t}\right]=\mathbb{E}\left[\left\{\tau_{0}^{1} \in B\right\} \mid \mathcal{F}^{\prime}{ }_{t}\right]$ almost surely on $\left\{J_{t}=1\right\}$. To see this, let $\epsilon>0$ define $\Lambda:=\left\{\omega \in \Omega: \mathbb{E}\left[\left\{\tau_{0}^{N_{t}+1} \in B\right\} \mid \mathcal{F}^{\prime}{ }_{t}\right]-\mathbb{E}\left[\left\{\tau_{0}^{1} \in\right.\right.\right.$ $\left.\left.B\} \mid \mathcal{F}^{\prime}{ }_{t}\right] \geq \epsilon\right\}$. This set is in $\mathcal{F}_{t}^{\prime}$, so by the above calculation we have that

$$
0=\int_{\Lambda \cap\left\{J_{t}=1\right\}} \mathbb{E}\left[\left\{\tau_{0}^{N_{t}+1} \in B\right\} \mid \mathcal{F}^{\prime}{ }_{t}\right]-\mathbb{E}\left[\left\{\tau_{0}^{1} \in B\right\} \mid \mathcal{F}^{\prime}{ }_{t}\right] d \mathbb{P} \geq \in \mathbb{P}\left(\Lambda \cap\left\{J_{t}=1\right\}\right) .
$$

So $\mathbb{P}\left(\Lambda \cap\left\{J_{t}=1\right\}\right)=0$. The same argument with $\Lambda^{\prime}:=\left\{\omega \in \Omega: \mathbb{E}\left[\left\{\tau_{0}^{1} \in\right.\right.\right.$ $\left.\left.B\} \mid \mathcal{F}_{t}^{\prime}\right]-\mathbb{E}\left[\left\{\tau_{0}^{N_{t}+1} \in B\right\} \mid \mathcal{F}_{t}^{\prime}\right] \geq \epsilon\right\}$ completes the proof of the claim. Therefore, $J_{t} \mathbb{E}\left[\left\{\tau_{0}^{N_{t}+1} \in B\right\} \mid \mathcal{F}_{t}^{\prime}\right]=J_{t} \mathbb{E}\left[\left\{\tau_{0}^{1} \in B\right\} \mid \mathcal{F}_{t}^{\prime}\right]$ almost surely.

Lemma 8. For each $t \geq 0$, define $\mathcal{F}_{t}^{\prime}$ to be the $\sigma$-algebra generated by $S_{N_{t}+1}^{\prime}$, $\tau_{1}^{N_{t}+1}$, and $\left\{\left(\tau_{0}^{k}, \tau_{1}^{k}\right)\right\}_{k=N_{t}+2}^{\infty}$. Then

$$
\mathbb{E}\left[\left\{\tau_{0}^{1} \in B\right\} \mid \mathcal{F}^{\prime}{ }_{t}\right] \rightarrow \mathbb{E}\left\{\tau_{0} \in B\right\} \quad \text { almost surely as } t \rightarrow \infty .
$$

Remark 6 . The proof of this Lemma is very similar to the proof of Lemma 6 .

Proof. Define $\mathcal{F}^{\prime}{ }_{\infty}:=\cap_{t \geq 0} \mathcal{F}_{t}^{\prime}$ and observe that $\mathcal{F}^{\prime}{ }_{s} \supset \mathcal{F}^{\prime}{ }_{t}$ for $0 \leq s \leq t$. Thus, by Lemma 2

$$
\mathbb{E}\left[\left\{\tau_{0}^{1} \in B\right\} \mid \mathcal{F}^{\prime}{ }_{t}\right] \rightarrow \mathbb{E}\left[\left\{\tau_{0}^{1} \in B\right\} \mid \mathcal{F}_{\infty}^{\prime}\right] \quad \text { almost surely. }
$$

We claim that for each $A \in \mathcal{F}_{\infty}^{\prime}, \mathbb{P}(A)=0$ or 1 . To show this, we will show that $\mathcal{F}_{\infty}^{\prime}$ is contained in the exchangeable $\sigma$-algebra and then apply the HewittSavage zero-one law. Let $n \in \mathbb{N}, A \in \mathcal{F}_{\infty}^{\prime}$, and $\pi_{n}$ be an arbitrary permutation of $\left(\tau_{0}^{1}, \tau_{1}^{1}\right), \ldots,\left(\tau_{0}^{n}, \tau_{1}^{n}\right)$.

Define $\pi_{t}: \Omega \rightarrow \Omega$ by

$$
\pi_{t}(\omega)= \begin{cases}\pi_{n}(\omega) & N_{t} \geq n \\ \omega & N_{t}<n .\end{cases}
$$

Since $S_{N_{t}+1}^{\prime}, \tau_{1}^{N_{t}+1}$, and $\left\{\left(\tau_{0}^{k}, \tau_{1}^{k}\right)\right\}_{k=N_{t}+2}^{\infty}$ are $\pi_{t}$-invariant, then $A$ is $\pi_{t}$-invariant by Lemma 4 as $A \in \mathcal{F}_{\infty}^{\prime} \subset \mathcal{F}_{t}^{\prime}$. Therefore

$$
\mathbb{P}\left(A \Delta \pi_{n}^{-1} A, N_{t} \geq n\right)=\mathbb{P}\left(A \Delta \pi_{t}^{-1} A, N_{t} \geq n\right) \leq \mathbb{P}\left(A \Delta \pi_{t}^{-1} A\right)=0 .
$$

Hence

$$
\begin{aligned}
\mathbb{P}\left(A \Delta \pi_{n}^{-1} A\right) & =\mathbb{P}\left(A \Delta \pi_{n}^{-1} A, N_{t} \geq n\right)+\mathbb{P}\left(A \Delta \pi_{n}^{-1} A, N_{t}<n\right) \\
& \leq \mathbb{P}\left(A \Delta \pi_{n}^{-1} A, N_{t}<n\right) \leq \mathbb{P}\left(N_{t}<n\right) .
\end{aligned}
$$

Since $t$ was arbitrary, we conclude that $\mathbb{P}\left(A \Delta \pi_{n}^{-1} A\right)=0$ because $\mathbb{P}\left(N_{t}<n+\right.$ 1) $\rightarrow 0$ as $t \rightarrow \infty$ since $N_{t} \rightarrow \infty$ almost surely. Since $\pi_{n}$ was an arbitrary finite permutation, we conclude that $\mathcal{F}_{\infty}^{\prime}$ is contained in the exchangeable $\sigma$-algebra. By 
the Hewitt-Savage zero-one law, $\mathcal{F}_{\infty}^{\prime}$ contains only events that have probability 0 or 1. Thus, $\left\{\tau_{0}^{1} \in C\right\}$ is trivially independent of $\mathcal{F}_{\infty}^{\prime}$ and so we conclude that $\mathbb{E}\left[\left\{\tau_{0}^{1} \in C\right\} \mid \mathcal{F}_{\infty}^{\prime}\right]=\mathbb{E}\left\{\tau_{0}^{1} \in C\right\}=\mathbb{E}\left\{\tau_{0} \in C\right\}$.

\section{PDES WITH RANDOMLY SWITCHING BOUNDARY CONDITIONS}

We now use our results from Section 2 to study parabolic PDEs with randomly switching boundary conditions. Our results apply to a range of specific problems, so in Section 3.1 we explain how to cast a problem in our framework. In Section 3.2 we collect assumptions and in Section 3.3 we prove theorems about the mean of the process.

3.1. General setup. Our results can be applied to the following type of random PDE. Suppose we are given a strongly elliptic, symmetric, second order differential operator $L$ on a domain $D \subset \mathbb{R}^{d}$ with smooth coefficients which do not depend on $t$. Assume the domain $D$ is bounded with a smooth boundary. We consider the stochastic process $u(t, x)$ that solves

$$
\partial_{t} u=L u \quad \text { in } D
$$

subject to boundary conditions that switch at random times between two given boundary conditions, $(a)$ and $(b)$. We allow $(a)$ and $(b)$ to be different types; for example, one can be Dirichlet and the other Neumann. For the sake of presentation, we assume $(a)$ are homogenous, but our analysis is easily modified to include the case where $(a)$ are inhomogenous.

We formulate this problem in the setting of Section 2 as alternating flows on the Hilbert space $L^{2}(D)$. We define

$$
A u:=L u \quad \text { if } u \in D(A) \quad \text { and } \quad B u:=L u \quad \text { if } u \in D(B)
$$

where $D(A)$ is chosen so that $A$ generates the contraction $C_{0}$-semigroup that maps an initial condition to the solution of Equation (13) at time $t$ subject to boundary conditions $(a)$, and $D(B)$ is chosen so that $B$ generates the contraction $C_{0^{-}}$ semigroup that maps an initial condition to the solution of Equation (13) at time $t$ subject to the homogenous version of boundary conditions $(b)$. We then choose $h(t):[0, \infty) \rightarrow D(L)$ to satisfy $\partial_{t} h=L h$ with boundary conditions $(b)$ and initial condition $h(0)=0$. Then the $H$-valued process defined in Equation (7) in Section2 with $\Phi_{t}^{1}(f)=e^{A t} f$ and $\Phi_{t}^{0}(f)=e^{B t} f+h(t)$ corresponds to this random PDE.

3.2. Assumptions. We now formalize the setup from Section 3.1 Let $H$ be a real separable Hilbert space with inner product $\langle\cdot, \cdot\rangle$ and let $A$ and $B$ be two self-adjoint operators on $H$, one with strictly negative spectrum and one with non-positive spectrum. Hence, $A$ and $B$ generate contraction $C_{0}$-semigroups, which we denote respectively by $e^{A t}$ and $e^{B t}$. Assume $A=B$ on $D(A) \cap D(B) \neq \emptyset$. Assume there exists a continuous function $h(t):[0, \infty) \rightarrow H$ satisfying $h(0)=0$ and $\frac{d}{d t}\langle\phi, h(t)\rangle=$ $\langle B \phi, h(t)\rangle$ for all $\phi \in D(A) \cap D(B)$. Recalling notation from Section 2.1, let the switching time distributions, $\mu_{0}$ and $\mu_{1}$, be continuous distributions on the positive real line.

Let $u(t, \omega)$ be the $H$-valued process defined in Equation (7) in Section 2.2 with

$$
\Phi_{t}^{1}(f)=e^{A t} f \quad \text { and } \quad \Phi_{t}^{0}(f)=e^{B t} f+h(t) .
$$

It's easy to check that $\Phi_{t}^{1}$ and $\Phi_{t}^{0}$ satisfy assumptions (a)-(e) from Section 2.1 . 
Assume there exists a deterministic $M=M\left(u_{0}\right)$ so that with probability one, $\|u(t)\| \leq M$ for each $t \geq 0$, where $\|x\|:=\sqrt{\langle x, x\rangle}$.

For every $0<s \leq t$, define $\eta(s, t)$ to be the random variable that gives the number of switches that occur on the interval $(s, t)$. Formally, we define $\eta(s, t)$ by taking the supremum over partitions $\sigma$ of the interval $(s, t), s=\sigma_{0}<\sigma_{1}<\cdots<$ $\sigma_{k}<\sigma_{k+1}=t$

$$
\eta(s, t)(\omega):=\sup _{\sigma} \sum_{i=0}^{k}\left|J_{\sigma_{i+1}}(\omega)-J_{\sigma_{i}}(\omega)\right|,
$$

where $J_{t}$ is as in Equation (6). Assume that $\mu_{0}$ and $\mu_{1}$ are such that for every $t>0$, we have that as $s \rightarrow 0$,

$$
\mathbb{P}(\eta(t, t+s)=1)=O(s) \text { and } \mathbb{P}(\eta(t, t+s) \geq 2)=o(s) .
$$

3.3. The mean satisfies the PDE. In what follows, fix $\phi \in D(A) \cap D(B)$, which will serve as our test function. The following theorem states that the mean of our process satisfies the weak form of the PDE.

Theorem 2. For each $\phi \in D(A) \cap D(B)$ and $t>0$, we have that

$$
\frac{d}{d t}\langle\phi, \mathbb{E} u(t)\rangle=\langle A \phi, \mathbb{E} u(t)\rangle
$$

To prove this theorem, we need a few lemmas. Our first lemma states that each realization our stochastic process satisfies the weak form of the PDE away from switching times.

Lemma 9. Let $\omega_{0} \in \Omega$ be given. If $t_{0}>0$ is such that $t_{0} \neq S_{k}\left(\omega_{0}\right)$ and $t_{0} \neq S_{k}^{\prime}\left(\omega_{0}\right)$ for every $k$, then for all $t$ in some neighborhood of $t_{0}$,

$$
\frac{d}{d t}\left\langle\phi, u\left(t, \omega_{0}\right)\right\rangle=\left\langle A \phi, u\left(t, \omega_{0}\right)\right\rangle .
$$

Proof. By the definition of $u(t, \omega)$ and the assumption that $A$ and $B$ are selfadjoint, we can write the inner product of $\phi$ and $u$ as

$$
\begin{aligned}
\langle\phi, u(t)\rangle & =\left\langle\phi, e^{A a_{t}} u\left(S_{N_{t}+1}^{\prime}\right)\right\rangle J_{t}+\left\langle\phi, e^{B a_{t}} u\left(S_{N_{t}}\right)+h\left(a_{t}\right)\right\rangle\left(1-J_{t}\right) \\
& =\left\langle e^{A a_{t}} \phi, u\left(S_{N_{t}+1}^{\prime}\right)\right\rangle J_{t}+\left\langle e^{B a_{t}} \phi, u\left(S_{N_{t}}\right)\right\rangle\left(1-J_{t}\right)+\left\langle\phi, h\left(a_{t}\right)\right\rangle\left(1-J_{t}\right) .
\end{aligned}
$$

We now calculate $\frac{d}{d t} e^{A a_{t}} \phi$ and $\frac{d}{d t} e^{B a_{t}} \phi$ where $\frac{d}{d t}$ means the limit in $H$ of the difference quotients. Since $t_{0}$ is such that $t_{0} \neq S_{k}\left(\omega_{0}\right)$ and $t_{0} \neq S_{k}^{\prime}\left(\omega_{0}\right)$ for all $k$, there exists a neighborhood $J\left(\omega_{0}\right)=J$ of $t_{0}$ so that no switches occur in $J$. Therefore $S_{N_{t}}, S_{N_{t}+1}^{\prime}$, and $J_{t}$ are constant on $J$. And since $e^{A t}$ is a $C_{0}$-semigroup and $\phi \in D(A)$, we have that for all $t \in J$

$$
\frac{d}{d t} e^{A a_{t}} \phi=\frac{d}{d t} e^{A\left(t-S_{N_{t}+1}^{\prime}\right)} \phi=\frac{d}{d t} e^{A t} e^{-A S_{N_{t}+1}^{\prime} \phi}=A e^{A t} e^{-A S_{N_{t}+1}^{\prime} \phi}=A e^{A a_{t}} \phi
$$

Similarly, $\frac{d}{d t} e^{B a_{t}} \phi=B e^{B a_{t}} \phi$. Since strongly convergent sequences in $H$ are weakly convergent, and again since $S_{N_{t}}, S_{N_{t}+1}^{\prime}$, and $J_{t}$ are constant on $J$, we have that for all $t \in J$

$$
\frac{d}{d t}\langle\phi, u(t)\rangle=\left\langle A e^{A a_{t}} \phi, u\left(S_{N_{t}+1}^{\prime}\right)\right\rangle J_{t}+\left(\left\langle B e^{B a_{t}} \phi, u\left(S_{N_{t}}\right)\right\rangle+\frac{d}{d t}\left\langle\phi, h\left(a_{t}\right)\right\rangle\right)\left(1-J_{t}\right) .
$$


Since $A$ and $B$ are self-adjoint, $A=B$ on $D(A) \cap D(B)$, and $\frac{d}{d t}\langle\phi, h(t)\rangle=(B \phi, h(t))$, we conclude that for all $t \in J$,

$$
\begin{aligned}
\frac{d}{d t}\langle\phi, u(t)\rangle & =\left\langle A \phi, e^{A a_{t}} u\left(S_{N_{t}+1}^{\prime}\right)\right\rangle J_{t}+\left\langle B \phi, e^{B a_{t}} u\left(S_{N_{t}}\right)+h\left(a_{t}\right)\right\rangle\left(1-J_{t}\right) \\
& =\langle A \phi, u(t)\rangle J_{t}+\langle B \phi, u(t)\rangle\left(1-J_{t}\right)=\langle A \phi, u(t)\rangle .
\end{aligned}
$$

The next lemma states that our process satisfies a weak continuity condition.

Lemma 10. For every $\epsilon>0$ and $t>0$, there exists a $\delta(\epsilon, t)>0$ so that if $|t-s|<\delta(\epsilon, t)$, then

$$
\left|\langle\phi, u(t, \omega)-u(s, \omega)\rangle 1_{\eta(s, t)=1}\right|<\epsilon \quad \text { a.s. }
$$

Proof. Let $s$ and $t$ be given and let $\rho$ be the minimum of $s$ and $t$. Observe that if there are no switches between $s$ and $t$ and $J_{\rho}=0$, then

$$
|\langle\phi, u(t, \omega)-u(s, \omega)\rangle|=\left|\left\langle\phi,\left[e^{A|t-s|}-I\right] u(\rho, \omega)\right\rangle\right| \leq\left\|e^{A|t-s|} \phi-\phi\right\| M,
$$

since $A$ is self-adjoint and $\|u(t)\| \leq M$ a.s. by assumption. Similarly, suppose there are no switches between $s$ and $t$ and $J_{\rho}=1$. If $M_{2}=\max _{\xi \leq 2 t}\|h(\xi)\|$ and $|t-s|<t$, then we have by the mean value theorem

$$
\begin{aligned}
\mid\langle\phi, u(t, \omega) & -u(s, \omega)\rangle|\leq|\left\langle\phi,\left[e^{B|t-s|}-I\right]\left[u(\rho, \omega)-h\left(a_{\rho}\right)\right]\right\rangle|+|\left\langle\phi, h\left(a_{t}\right)-h\left(a_{s}\right)\right\rangle \mid \\
& \leq\left|\left\langle\left[e^{B|t-s|}-I\right] \phi, u(\rho, \omega)-h\left(a_{\rho}\right)\right\rangle\right|+|t-s| \max _{\xi \leq 2 t} \frac{d}{d t}|\langle\phi, h(\xi)\rangle| \\
& \leq\left\|e^{B|t-s|} \phi-\phi\right\|\left(M+M_{2}\right)+|t-s||| B \phi \| M_{2}
\end{aligned}
$$

Since $e^{A t}$ and $e^{B t}$ are both $C_{0}$-semigroups, we can choose a $0<\delta(\epsilon, t)<t$ so that if $|t-s|<\delta(\epsilon, t)$, then

$$
\max \left\{\left\|e^{A|t-s|} \phi-\phi\right\|,\left\|e^{B|t-s|} \phi-\phi\right\|,|t-s|\right\}<\frac{\epsilon}{M+M_{2}+\|B \phi\| M_{2}} .
$$

Let $\omega \in \Omega$ be given and assume $|t-s|<\delta(\epsilon, t)$. If $\omega$ is such that $\eta(s, t)(\omega) \neq 1$, then the result is immediate. Suppose $\eta(s, t)(\omega)=1$. If $\sigma$ denotes the switching time between $s$ and $t$, then

$|\langle\phi, u(t, \omega)-u(s, \omega)\rangle| \leq|\langle\phi, u(t, \omega)-u(\sigma, \omega)\rangle|+|\langle\phi, u(\sigma, \omega)-u(s, \omega)\rangle|<3 \epsilon$.

Proof of Theorem 圆. We seek to differentiate $\mathbb{E}\langle\phi, u(t)\rangle$ with respect to $t$. Define

$$
f(t, \omega)=\langle\phi, u(t, \omega)\rangle .
$$

Let $h_{n} \rightarrow 0$ as $n \rightarrow \infty$. For a given $t_{0}>0$, define the difference quotient

$$
\begin{aligned}
g_{n}(\omega) & :=\frac{1}{h_{n}}\left(f\left(t_{0}+h_{n}, \omega\right)-f\left(t_{0}, \omega\right)\right) \\
& =g_{n}(\omega) 1_{\eta\left(t_{0}+h_{n}, t_{0}\right)=0}+g_{n}(\omega) 1_{\eta\left(t_{0}+h_{n}, t_{0}\right)=1}+g_{n}(\omega) 1_{\eta\left(t_{0}+h_{n}, t_{0}\right) \geq 2} \\
& =(1)+(2)+(3),
\end{aligned}
$$

where $\eta$ is defined in Equation (14). We will handle each of these terms differently.

We first consider (1). Assume $\omega$ is such that $t_{0}$ is not a switching time. By Lemma 9 .

$$
\frac{1}{h_{n}}\left(f\left(t_{0}+h_{n}, \omega\right)-f\left(t_{0}, \omega\right)\right) \rightarrow \frac{d}{d t} f\left(t_{0}, \omega\right)=\left\langle A \phi, u\left(t_{0}\right)\right\rangle \text { as } n \rightarrow \infty .
$$


Also observe that for such an $\omega$, we have that $1_{\eta\left(t_{0}+h_{n}, t_{0}\right)=0}(\omega)=1$ for $n$ sufficiently large. Since $\mu_{0}$ and $\mu_{1}$ are continuous distributions, this set of $\omega$ 's has probability 1 , and thus

$$
(1)=\frac{1}{h_{n}}\left(f\left(t_{0}+h_{n}, \omega\right)-f\left(t_{0}, \omega\right)\right) 1_{\eta\left(t_{0}+h_{n}, t_{0}\right)=0} \rightarrow\left\langle A \phi, u\left(t_{0}\right)\right\rangle \quad \text { a.s. as } n \rightarrow \infty .
$$

We now apply the bounded convergence theorem to (1). Let $n$ and $\omega$ be given. If $\eta\left(t_{0}+h_{n}, t_{0}\right)(\omega) \neq 0$, then $|(1)|=0$, trivially. If $\eta\left(t_{0}+h_{n}, t_{0}\right)(\omega)=0$, then $f(t, \omega)$ is differentiable in $t$ for all $t \in\left(t_{0}, t_{0}+h_{n}\right)$. Therefore we can employ the mean value theorem to obtain

$$
\begin{aligned}
\left|\frac{1}{h_{n}}\left(f\left(t_{0}+h_{n}, \omega\right)-f\left(t_{0}, \omega\right)\right)\right| & \leq \sup _{t \in\left(t_{0}, t_{0}+h_{n}\right)}\left|\frac{d}{d t} f(t, \omega)\right| \\
& =\sup _{t \in\left(t_{0}, t_{0}+h_{n}\right)}|\langle A \phi, u(t, \omega)\rangle| \leq\|A \phi\| M,
\end{aligned}
$$

since $\|u(t)\| \leq M$ by assumption. Thus $|(1)| \leq\|A \phi\| M$ almost surely and so by the bounded convergence theorem, $\mathbb{E}(1) \rightarrow \mathbb{E}\left\langle A \phi, u\left(t_{0}\right)\right\rangle$ as $n \rightarrow \infty$.

To complete the proof, we need only show that (2) and (3) both tend to 0 in mean as $n \rightarrow \infty$. We first work on (2). Observe that

$$
\begin{aligned}
\mathbb{E}|(2)| & =\mathbb{E}\left|\frac{1}{h_{n}}\left(f\left(t_{0}+h_{n}, \omega\right)-f\left(t_{0}, \omega\right)\right) 1_{\eta\left(t_{0}+h_{n}, t_{0}\right)=1}\right| \\
& \leq \frac{1}{h_{n}} \operatorname{ess}^{\sup _{\omega}}\left|\left(f\left(t_{0}+h_{n}, \omega\right)-f\left(t_{0}, \omega\right)\right) 1_{\eta\left(t_{0}+h_{n}, t_{0}\right)=1}\right| \mathbb{E}\left(1_{\eta\left(t_{0}+h_{n}, t_{0}\right)=1}\right) .
\end{aligned}
$$

It follows from Lemma 10 that $\operatorname{ess} \sup _{\omega}\left|\left(f\left(t_{0}+h_{n}, \omega\right)-f\left(t_{0}, \omega\right)\right) 1_{\eta\left(t_{0}+h_{n}, t_{0}\right)=1}\right| \rightarrow$ 0 as $n \rightarrow \infty$. Since by assumption $\mathbb{P}\left(\eta\left(t_{0}+h_{n}, t_{0}\right)=1\right)=O\left(h_{n}\right)$, we conclude that $\mathbb{E}|(2)| \rightarrow 0$ as $n \rightarrow \infty$.

Finally, we consider (3). By the assumption that $\|u(t)\| \leq M$,

$$
\begin{aligned}
\mathbb{E}|(3)| & =\mathbb{E}\left|\frac{1}{h_{n}}\left(f\left(t_{0}+h_{n}, \omega\right)-f\left(t_{0}, \omega\right)\right) 1_{\eta\left(t_{0}+h_{n}, t_{0}\right) \geq 2}\right| \\
& \leq \frac{2\|\phi\| M}{h_{n}} \mathbb{P}\left(\eta\left(t_{0}+h_{n}, t_{0}\right) \geq 2\right) .
\end{aligned}
$$

By assumption, $\mathbb{P}\left(\eta\left(t_{0}+h_{n}, t_{0}\right) \geq 2\right)=o\left(h_{n}\right)$, and hence $\mathbb{E}|(3)| \rightarrow 0$ as $n \rightarrow \infty$.

Therefore

$$
\frac{\mathbb{E}\left\langle\phi, u\left(t_{0}+h_{n}\right)\right\rangle-\mathbb{E}\left\langle\phi, u\left(t_{0}\right)\right\rangle}{h_{n}}=\mathbb{E} g_{n} \rightarrow \mathbb{E}\left\langle A \phi, u\left(t_{0}\right)\right\rangle \text { as } n \rightarrow \infty .
$$

Since $h_{n}$ was an arbitrary sequence tending to 0 and $t_{0}$ was an arbitrary positive number, we conclude that $\frac{d}{d t} \mathbb{E}\langle\phi, u(t)\rangle=\mathbb{E}\langle A \phi, u(t)\rangle$ for all $t>0$.

Since taking the inner product against $\phi$ or $A \phi$ are both bounded linear operators on $H$, we can exchange expectation with inner product to obtain

$$
\frac{d}{d t}\langle\phi, \mathbb{E} u(t)\rangle=\frac{d}{d t} \mathbb{E}\langle\phi, u(t)\rangle=\mathbb{E}\langle A \phi, u(t)\rangle=\langle A \phi, \mathbb{E} u(t)\rangle .
$$

We now show that the mean at large time satisfies the homogeneous PDE.

Theorem 3. Let $\phi \in D(A) \cap D(B)$. Then $\mathbb{E} u(t) \rightarrow \mathbb{E} \bar{u}$ weakly in $H$ as $t \rightarrow \infty$, where $\bar{u}$ is as in Theorem 1 . Furthermore, $\mathbb{E} \bar{u}$ satisfies

$$
\langle A \phi, \mathbb{E} \bar{u}\rangle=0 \text {. }
$$


Remark 7. We will often assume the differential operator and the domain to be sufficiently regular so that this theorem implies $\mathbb{E} \bar{u}$ is a $C^{\infty}$ function satisfying the PDE pointwise.

Proof. Since the switching time distributions, $\mu_{0}$ and $\mu_{1}$, are assumed to be continuous distributions, they are non-arithmetic. Hence, by Theorem $1 \mathbb{E} g(u(t)) \rightarrow$ $\mathbb{E} g(\bar{u}(t))$ as $t \rightarrow \infty$ for every continuous and bounded $g: H \rightarrow \mathbb{R}$. For any $\eta \in H$, the function $(\eta, \cdot): H \rightarrow \mathbb{R}$ is continuous and since by assumption, $\|u(t)\| \leq M$ a.s., it follows that

$$
\mathbb{E}\langle\eta, u(t)\rangle \rightarrow \mathbb{E}\langle\eta, \bar{u}\rangle \quad \text { as } t \rightarrow \infty .
$$

Since taking the inner product against $\eta$ is a bounded linear operator on $H$, we can exchange expectation with inner product in Equation (15) above. Hence, $\mathbb{E} u(t) \rightarrow$ $\mathbb{E} \bar{u}$ weakly in $H$ as $t \rightarrow \infty$.

Of course it follows that in particular

$$
\langle\phi, \mathbb{E} u(t)\rangle \rightarrow\langle\phi, \mathbb{E} \bar{u}\rangle \quad \text { and } \quad\langle A \phi, \mathbb{E} u(t)\rangle \rightarrow\langle A \phi, \mathbb{E} \bar{u}\rangle \quad \text { as } t \rightarrow \infty .
$$

By Theorem $2 \frac{d}{d t}\langle\phi, \mathbb{E} u(t)\rangle=\langle A \phi, \mathbb{E} u(t)\rangle$. Thus, $\langle\phi, \mathbb{E} u(t)\rangle$ and $\frac{d}{d t}\langle\phi, \mathbb{E} u(t)\rangle$ both converge as $t \rightarrow \infty$ and so we conclude that $\frac{d}{d t}\langle\phi, \mathbb{E} u(t)\rangle$ must actually converge to 0 . Hence, $\langle A \phi, \mathbb{E} \bar{u}\rangle=0$.

\section{EXAMPLES}

In this section we apply our results from Sections 2 and 3 to the heat equation on the interval $[0, L]$. We impose an absorbing Dirichlet boundary condition at $x=0$ and a stochastically switching boundary condition at $x=L$. In Example 1 we consider switching between a Dirichlet and a Neumann boundary condition at $x=L$. In Example 2, we consider switching between two Dirichlet boundary conditions at $x=L$.

4.1. Example 1; Dirichlet/Neumann switching. Consider the stochastic process that solves

$$
\partial_{t} u=D \Delta u \quad \text { in }(0, L)
$$

and at exponentially distributed times switches between the boundary conditions

$$
\left\{\begin{array} { l } 
{ u ( 0 , t ) = 0 } \\
{ u _ { x } ( L , t ) = 0 }
\end{array} \text { and } \left\{\begin{array}{l}
u(0, t)=0 \\
u(L, t)=b>0 .
\end{array}\right.\right.
$$

To cast this problem in the setting of previous sections, we set our Hilbert space to be $L^{2}[0, L]$ and define the operators

$$
\begin{array}{ll}
A u:=\Delta u & \text { if } u \in D(A):=\left\{\phi \in H^{2}(0, L): \frac{\partial \phi}{\partial \mathbf{n}}(L)=0=\phi(0)\right\} \\
B u:=\Delta u & \text { if } u \in D(B):=H_{0}^{1}(0, L) \cap H^{2}(0, L) .
\end{array}
$$

We set $c=\frac{b}{L} x \in L^{2}[0, L]$ and let our switching time distributions, $\mu_{0}$ and $\mu_{1}$, be exponential with respective rate parameters $r_{0}$ and $r_{1}$. Let $u(t, \omega)$ be the $H$-valued process defined in Equation (7) with

$$
\Phi_{t}^{1}(f)=e^{A t} f \quad \text { and } \quad \Phi_{t}^{0}(f)=e^{B t}(f-c)+c .
$$

We are interested in studying the large time distribution of $u(t)$. By Corollary 1 we have that $u(t)$ converges in distribution as $t \rightarrow \infty$ to the $L^{2}[0, L]$-valued random 
variable $\bar{u}$ defined in the statement of the corollary. By the definitions of $Y_{0}$ and $Y_{1}$ in Equation (3), it is immediate that $\bar{u}$ is almost surely smooth, and using Proposition [3, it follows that $\bar{u}(x) \leq \frac{b}{L} x$ almost surely for each $x \in[0, L]$. In this section, we will find the expectation of $\bar{u}$.

Proposition 4. The function $\mathbb{E} \bar{u}$ is affine with slope

$$
\left(1+\frac{\rho}{\gamma} \tanh (\gamma)\right)^{-1} \frac{b}{L}
$$

where $\gamma=L \sqrt{\left(r_{0}+r_{1}\right) / D}$ and $\rho=r_{0} / r_{1}$.

To prove this proposition, we will use the results from both Sections 2 and 3 It is immediate that all of the assumptions in Section 3.2 are satisfied, except for one; we need to check that there exists a deterministic $M$ so that $\|u(t)\| \leq M$ almost surely for all $t \geq 0$. We show that and more in the following lemma.

Lemma 11. Under the assumptions of the current section, we have that

$$
\|u(t)\| \leq L\left(\max \left\{\left\|u_{0}\right\|_{\infty}, b\right\}\right)^{2},
$$

where $\|\cdot\|_{\infty}$ denotes the $L^{\infty}[0, L]$ norm. Furthermore,

$$
\left\|Y_{1}\right\|_{\infty} \leq b \quad \text { and } \quad\left\|Y_{0}\right\|_{\infty} \leq b \quad \text { almost surely. }
$$

Proof. First note that $\|c\|_{\infty}=\left\|\frac{b}{L} x\right\|_{\infty}=b$. If $f \in L^{2}[0, L]$, then by the maximum principle, we have that for any $t \geq 0$

$$
\left\|e^{A t} f\right\|_{\infty} \leq\|f\|_{\infty} \text { and }\left\|e^{B t}(f-c)+c\right\|_{\infty} \leq \max \left\{b,\|f\|_{\infty}\right\} .
$$

Hence, $\max \left\{\|u(t)\|_{\infty}, b\right\}$ is non-increasing in $t$ and so the bound on $\|u(t)\|$ is proven.

Since $S:=\left\{f \in L^{2}[0, L]:\|f\|_{\infty} \leq b\right\}$ is a closed set in $L^{2}[0, L]$, Equation (19) and Proposition 3 give the desired bounds on $\left\|Y_{1}\right\|_{\infty}$ and $\left\|Y_{0}\right\|_{\infty}$.

As in Corollary 1 let $\bar{u}$ have the limiting distribution of $u(t)$ as $t \rightarrow \infty$. Then by Theorem [3, we have that $\mathbb{E} \bar{u} \in L^{2}[0, L]$ satisfies $\langle\Delta \phi, \mathbb{E} \bar{u}\rangle=0$ for each $\phi \in C_{0}^{\infty}(0, L)$. By the regularity of $\Delta$ on $[0, L]$, it follows that $\mathbb{E} \bar{u}$ is the affine function

$$
(\mathbb{E} \bar{u})(x)=s x+d
$$

for some $s, d \in \mathbb{R}$. By Corollary 1 of Section 2, we have that

$$
s x+d=p \mathbb{E} Y_{1}+(1-p) \mathbb{E} Y_{0}
$$

where $p=r_{0} /\left(r_{0}+r_{1}\right)$. We will use Equation (20) to determine $s$ and $d$. While both $Y_{0}$ and $Y_{1}$ are almost surely smooth functions, $\mathbb{E} Y_{0}$ and $\mathbb{E} Y_{1}$ are a priori only elements of $L^{2}[0, L]$. It can be shown that $\mathbb{E} Y_{0}$ and $\mathbb{E} Y_{1}$ are smooth functions, but we will instead take limits of test functions to avoid evaluating $\mathbb{E} Y_{0}$ and $\mathbb{E} Y_{1}$ at specific points in $[0, L]$.

Let $\left\{\phi_{n}\right\}_{n=1}^{\infty}$ be such that $\phi_{n} \in C_{0}^{\infty}(0, L)$ and $\left\|\phi_{n}\right\|_{L^{1}}=1$ for each $n$ and

$$
\lim _{n \rightarrow \infty}\left\langle\phi_{n}, f\right\rangle=f(0)
$$

for each $f \in C[0, L]$. Since the inner product with $\phi_{n}$ is a bounded linear transformation in $L^{2}[0, L]$, we can interchange expectation with inner product in Equation (20) to obtain

$$
d=\lim _{n \rightarrow \infty}\left[\left\langle\phi_{n}, p \mathbb{E} Y_{1}+(1-p) \mathbb{E} Y_{0}\right\rangle\right]=\lim _{n \rightarrow \infty}\left[p \mathbb{E}\left\langle\phi_{n}, Y_{1}\right\rangle+(1-p) \mathbb{E}\left\langle\phi_{n}, Y_{0}\right\rangle\right]
$$


We want to exchange the limit with the expectations. To do this, first observe that $Y_{1}(x)$ and $Y_{0}(x)$ are each almost surely continuous functions of $x \in[0, L]$ with $Y_{0}(0)=0=Y_{1}(0)$ almost surely. Thus,

$$
\lim _{n \rightarrow \infty}\left\langle\phi_{n}, Y_{0}\right\rangle=0 \quad \text { and } \quad \lim _{n \rightarrow \infty}\left\langle\phi_{n}, Y_{1}\right\rangle=0 \quad \text { almost surely. }
$$

Using Lemma 11 and the assumption that $\left\|\phi_{n}\right\|_{L^{1}}=1$ for each $n$, we have that

$$
\left|\left\langle\phi_{n}, Y_{0}\right\rangle\right| \leq b \quad \text { and } \quad\left|\left\langle\phi_{n}, Y_{1}\right\rangle\right| \leq b \quad \text { almost surely. }
$$

So we apply the bounded convergence theorem to Equation (21) to obtain

$$
d=p \mathbb{E} \lim _{n \rightarrow \infty}\left\langle\phi_{n}, Y_{1}\right\rangle+(1-p) \mathbb{E} \lim _{n \rightarrow \infty}\left\langle\phi_{n}, Y_{0}\right\rangle=0 .
$$

We now find the slope $s$ of $\mathbb{E} \bar{u}$. Denote the orthonormal eigenbasis of $A$ by $\left\{a_{k}\right\}_{k=1}^{\infty}$ and corresponding eigenvalues by $\left\{-\alpha_{k}\right\}_{k=1}^{\infty}$. Since $\sum_{k=1}^{n}\left\langle a_{k}, \mathbb{E} Y_{1}\right\rangle a_{k}$ converges to $\mathbb{E} Y_{1}$ in $L^{2}[0, L]$ as $n \rightarrow \infty$, we have that for any $\phi \in C_{0}^{\infty}(0, L)$

$$
\langle\phi, s x\rangle=\left\langle\phi, p \mathbb{E} Y_{1}\right\rangle+(1-p)\left\langle\phi, \mathbb{E} Y_{0}\right\rangle=p\left\langle\phi, \sum_{k=1}^{\infty}\left\langle a_{k}, \mathbb{E} Y_{1}\right\rangle a_{k}\right\rangle+(1-p)\left\langle\phi, \mathbb{E} Y_{0}\right\rangle .
$$

We will need the following lemma which is an immediate corollary of Proposition 2

Lemma 12. Under the assumptions of Section 4.1, we have that for each $k \in \mathbb{N}$

$$
\mathbb{E}\left[e^{-\alpha_{k} \tau_{1}}\right]\left\langle a_{k}, \mathbb{E} Y_{0}\right\rangle=\left\langle a_{k}, \mathbb{E} Y_{1}\right\rangle .
$$

Combining this lemma with $s x=p \mathbb{E} Y_{1}+(1-p) \mathbb{E} Y_{0}$ and rearranging terms yields

$$
\left\langle a_{k}, \mathbb{E} Y_{1}\right\rangle=\mathbb{E}\left[e^{-\alpha_{k} \tau_{1}}\right] \frac{s\left\langle a_{k}, x\right\rangle}{p \mathbb{E}\left[e^{-\alpha_{k} \tau_{1}}\right]+(1-p)} .
$$

Plugging this into Equation (22) gives

$$
\langle\phi, s x\rangle=p\left\langle\phi, \sum_{k=1}^{\infty} \mathbb{E}\left[e^{-\alpha_{k} \tau_{1}}\right] \frac{s\left\langle a_{k}, x\right\rangle}{p \mathbb{E}\left[e^{-\alpha_{k} \tau_{1}}\right]+(1-p)} a_{k}\right\rangle+(1-p)\left\langle\phi, \mathbb{E} Y_{0}\right\rangle
$$

Solving for $s$, we find that

$$
s=(1-p)\left\langle\phi, \mathbb{E} Y_{0}\right\rangle\left(\langle\phi, x\rangle-p\left\langle\phi, \sum_{k=1}^{\infty} \mathbb{E}\left[e^{-\alpha_{k} \tau_{1}}\right] \frac{\left\langle a_{k}, x\right\rangle}{p \mathbb{E}\left[e^{-\alpha_{k} \tau_{1}}\right]+(1-p)} a_{k}\right\rangle\right)^{-1}
$$

Let $\left\{\phi_{n}\right\}_{n=1}^{\infty} \in C_{0}^{\infty}(0, L)$ be such that $\left\|\phi_{n}\right\|_{L^{1}}=1$ for each $n$ and $\lim _{n \rightarrow \infty}\left\langle\phi_{n}, f\right\rangle=$ $f(L)$ for each $f \in C[0, L]$. Observe that $\lim _{n \rightarrow \infty}\left\langle\phi_{n}, x\right\rangle=L$ and using Lemma 11 and the same argument as above, we have that

$$
\lim _{n \rightarrow \infty}\left\langle\phi_{n}, \mathbb{E} Y_{0}\right\rangle=b
$$

Now, we want to show that

$$
\lim _{n \rightarrow \infty}\left\langle\phi_{n}, \sum_{k=1}^{\infty} \frac{\mathbb{E}\left[e^{-\alpha_{k} \tau_{1}}\right]\left\langle a_{k}, x\right\rangle}{p \mathbb{E}\left[e^{-\alpha_{k} \tau_{1}}\right]+(1-p)} a_{k}\right\rangle=\sum_{k=1}^{\infty} \frac{\mathbb{E}\left[e^{-\alpha_{k} \tau_{1}}\right]\left\langle a_{k}, x\right\rangle}{p \mathbb{E}\left[e^{-\alpha_{k} \tau_{1}}\right]+(1-p)} a_{k}(L) .
$$


To do this, we need to show that $\sum_{k=1}^{\infty} \frac{\mathbb{E}\left[e^{-\alpha_{k} \tau_{1}}\right]\left\langle a_{k}, x\right\rangle}{p \mathbb{E}\left[e^{-\alpha_{k} \tau_{1}}\right]+(1-p)} a_{k}(x)$ converges uniformly in $x$. Note that for each $k$

$$
a_{k}(x)=\sqrt{\frac{2}{L}} \sin \left(\frac{(2 k-1) \pi x}{2 L}\right) \quad \text { and } \quad \alpha_{k}=\frac{D(2 k-1)^{2} \pi^{2}}{4 L^{2}} .
$$

Hence, $\mathbb{E}\left[e^{-\alpha_{k} \tau_{1}}\right] \leq 1$ and $p \mathbb{E} e^{-\alpha_{k} \tau_{1}}+(1-p) \geq 1-p$. Furthermore,

$$
\left\|a_{k}\right\|_{\infty} \leq \sqrt{\frac{2}{L}} \quad \text { and } \quad\left\langle a_{k}, x\right\rangle=\frac{4 \sqrt{2} L^{3 / 2}}{\pi^{2}} \frac{(-1)^{k+1}}{(2 k-1)^{2}} .
$$

So for any $N \in \mathbb{N}$

$$
\begin{aligned}
\left\|\sum_{k=N}^{\infty} \frac{\mathbb{E}\left[e^{-\alpha_{k} \tau_{1}}\right]\left\langle a_{k}, x\right\rangle}{p \mathbb{E}\left[e^{-\alpha_{k} \tau_{1}}\right]+(1-p)} a_{k}(x)\right\|_{\infty} & \leq \sum_{k=N}^{\infty} \frac{\left|\left\langle a_{k}, x\right\rangle\right|}{1-p}\left\|a_{k}(x)\right\|_{\infty} \\
& =\sum_{k=N}^{\infty} \frac{16 L}{(1-p) \pi^{2}(2 k-1)^{2}} \rightarrow 0 \text { as } N \rightarrow \infty .
\end{aligned}
$$

Hence Equation (24) is verified, and thus by Equation (23) we have that

$$
s=\frac{(1-p) b}{L-p \sum_{k=1}^{\infty} \mathbb{E}\left[e^{-\alpha_{k} \tau_{1}}\right] \frac{\left\langle a_{k}, x\right\rangle}{p \mathbb{E} e^{-\alpha_{k} \tau_{1}+(1-p)}} a_{k}(L)} .
$$

Using the assumptions on $\tau_{0}, \tau_{1}, \alpha_{k}$, and $a_{k}$, and using a series simplification formula found in Mathematica ([4]), this becomes

$$
s=\left(1+\frac{\rho}{\gamma} \tanh (\gamma)\right)^{-1} \frac{b}{L}
$$

where $\gamma=L \sqrt{\left(r_{0}+r_{1}\right) / D}$ and $\rho=r_{0} / r_{1}$. This expectation is much different than the expectation we obtain when switching between boundary conditions of the same type in the next example below.

4.2. Example 2; Dirichlet/Dirichlet switching. Consider the stochastic process that solves

$$
\partial_{t} u=D \Delta u \quad \text { in }(0, L)
$$

and at exponentially distributed times switches between the boundary conditions

$$
\left\{\begin{array} { l } 
{ u ( 0 , t ) = 0 } \\
{ u ( L , t ) = 0 }
\end{array} \quad \text { and } \quad \left\{\begin{array}{l}
u(0, t)=0 \\
u(L, t)=b>0 .
\end{array}\right.\right.
$$

To cast this problem in the setting of previous sections, we set our Hilbert space to be $L^{2}[0, L]$ and define the operator

$$
B u:=\Delta u \quad \text { if } u \in D(B):=H_{0}^{1}(0, L) \cap H^{2}(0, L) .
$$

We set $c=\frac{b}{L} x \in L^{2}[0, L]$. Let our switching time distributions, $\mu_{0}$ and $\mu_{1}$, be exponential with respective rate parameters $r_{0}$ and $r_{1}$. Let $u(t, \omega)$ be the $H$-valued process defined in Equation (7) with

$$
\Phi_{t}^{1}(f)=e^{B t} f \quad \text { and } \quad \Phi_{t}^{0}(f)=e^{B t}(f-c)+c .
$$

We are interested in studying the large time distribution of $u(t)$. As in Example1, we can use Corollary 1 to obtain that $u(t)$ converges in distribution as $t \rightarrow \infty$ to some $L^{2}[0, L]$-valued random variable $\bar{u}$ defined in the statement of the corollary, 
and use Proposition 3 to obtain that $\bar{u}(x) \leq \frac{b}{L} x$ almost surely for each $x \in[0, L]$. And as in Example 1, we can use Theorem 3 to find the expectation of $\bar{u}$. However, since this problem switches between boundary conditions of the same type, we will be able to obtain much more information about $\bar{u}$.

Switching between two boundary conditions of the same type is significantly simpler than switching between boundary conditions of different types. This is because the two solution operators that we use when switching between boundary conditions of the same type both employ the same semigroup and thus the same orthonormal eigenbasis. Hence, we only need to consider the projections of the stochastic process in this one basis. In this example, the orthonormal eigenbasis and corresponding eigenvalues for $B$ are for $k \in \mathbb{N}$

$$
b_{k}=\sqrt{\frac{2}{L}} \sin \left(\frac{k \pi}{L} x\right) \quad \text { and } \quad-\beta_{k}=-D(k \pi / L)^{2} .
$$

Observe that for each $k$, the Fourier coefficient $u_{k}(t):=\left\langle b_{k}, u(t)\right\rangle \in \mathbb{R}$ is the solution to a one-dimensional ODE with a randomly switching right-hand side. Specifically, if $J_{t}$ is the jump process defined in Equation (6), then in between jumps of $J_{t}$ the process $u_{k}(t)$ satisfies

$$
\begin{aligned}
\frac{d}{d t} u_{k} & =-J_{t} \beta_{k} u_{k}-\left(1-J_{t}\right) \beta_{k}\left(u_{k}-c_{k}\right), \\
\text { where } \quad c_{k} & :=\left\langle b_{k}, c\right\rangle=\frac{(-1)^{k+1} b \sqrt{2 L}}{k \pi} .
\end{aligned}
$$

We can use previous results on one-dimensional ODEs with randomly switching right-hand sides (see [23] or [8]) to determine the marginal distributions of the Fourier coefficients of the stationary $\bar{u}$. For each $k$, the marginal distributions of the Fourier coefficients of $Y_{0}$ and $Y_{1}$ are given by

$$
\frac{\left\langle b_{k}, Y_{0}\right\rangle}{c_{k}} \sim \operatorname{Beta}\left(\frac{r_{1}}{\beta_{k}}+1, \frac{r_{0}}{\beta_{k}}\right) \quad \text { and } \quad \frac{\left\langle b_{k}, Y_{1}\right\rangle}{c_{k}} \sim \operatorname{Beta}\left(\frac{r_{1}}{\beta_{k}}, \frac{r_{0}}{\beta_{k}}+1\right) .
$$

Combining this with Corollary 1 gives the marginal distributions of the Fourier coefficients of $\bar{u}$.

From Equation (29) and Corollary 1, we obtain

$$
\mathbb{E} \bar{u}=(1-p) \frac{b}{L} x
$$

where $p=r_{0} /\left(r_{0}+r_{1}\right)$. Thus, the expectation of the process at large time is merely the solution to the time homogeneous PDE with boundary conditions given by the average of the two boundary conditions that the process switches between.

To further illustrate the usefulness of Equation (29), we calculate the $L^{2}$-variance of $\bar{u}$. It follows from Equation (30) that

$$
\mathbb{E}\|\bar{u}-\mathbb{E} \bar{u}\|^{2}=\mathbb{E}\|\bar{u}\|^{2}-\frac{L}{3} b^{2}(1-p)^{2} .
$$

Now by Corollary 1 , we have that $\mathbb{E}\|\bar{u}\|^{2}=p \mathbb{E}\left\|Y_{1}\right\|^{2}+(1-p) \mathbb{E}\left\|Y_{0}\right\|^{2}$. Combining this with Equation (29) we obtain

$$
\mathbb{E}\|\bar{u}\|^{2}=\sum_{k=1}^{\infty} \frac{r_{1}\left(r_{1}+\beta_{k}\right)}{\left(r_{0}+r_{1}\right)\left(r_{0}+r_{1}+\beta_{k}\right)} c_{k}^{2} .
$$


After plugging in our values for $\beta_{k}, b_{k}$ and $c_{k}$ in Equation (32), using a series simplification formula found in Mathematica (41]), and combining with Equation (31), we obtain the $L^{2}$-variance

$$
\mathbb{E}\|\bar{u}-\mathbb{E} \bar{u}\|^{2}=\frac{b^{2} D r_{1} r_{0}(\gamma \operatorname{coth}(\gamma)-1)}{L\left(r_{0}+r_{1}\right)^{3}},
$$

where $\gamma=L \sqrt{r_{0}+r_{1} / D}$.

While Equation (29) is useful, knowing the marginal distributions of the individual Fourier coefficients of $Y_{0}$ or $Y_{1}$ is of course not enough to find their joint distributions, and the one-dimensional ODE methods used to obtain Equation (29) do not give information about these joint distributions. We can, however, use our machinery developed in Section 2 to study these joint distributions.

First, we can use Corollary 1 and Proposition 2 to obtain joint statistics of the components of $\bar{u}$. To illustrate, we will calculate $\mathbb{E}\left\langle Y_{0}, b_{n}\right\rangle\left\langle Y_{0}, b_{m}\right\rangle$. Proposition 2 gives

$$
\mathbb{E}\left\langle Y_{0}, b_{n}\right\rangle\left\langle Y_{0}, b_{m}\right\rangle=\mathbb{E}\left\langle e^{B \tau_{0}}\left(e^{B \tau_{1}} Y_{0}-c\right)+c, b_{n}\right\rangle\left\langle e^{B \tau_{0}}\left(e^{B \tau_{1}} Y_{0}-c\right)+c, b_{m}\right\rangle,
$$

where $\tau_{0}$ and $\tau_{1}$ are independent exponential random variables with rates $r_{0}$ and $r_{1}$. After recalling some basic facts about exponential random variables and making some algebraic manipulations, we obtain that $\mathbb{E}\left\langle Y_{0}, b_{n}\right\rangle\left\langle Y_{0}, b_{m}\right\rangle$ is equal to

$$
\frac{\left(\beta_{m}+\beta_{n}+r_{1}\right)\left(\left(\beta_{m}+\beta_{n}\right)\left(\beta_{m}+r_{1}\right)\left(\beta_{n}+r_{1}\right)+\left(2 \beta_{m} \beta_{n}+\left(\beta_{m}+\beta_{n}\right) r_{1}\right) r_{0}\right)}{\left(\beta_{m}+\beta_{n}\right)\left(\beta_{m}+r_{1}+r_{0}\right)\left(\beta_{n}+r_{1}+r_{0}\right)\left(\beta_{m}+\beta_{n}+r_{1}+r_{0}\right)} c_{m} c_{n} .
$$

From this, we can readily compute the covariance of $\left\langle Y_{0}, b_{n}\right\rangle$ and $\left\langle Y_{0}, b_{m}\right\rangle$. Other joint statistics of the Fourier coefficients of $Y_{0}$ and $Y_{1}$ (and hence $\bar{u}$ by Corollary 1) are found in analogous ways.

Next, we can use Proposition 3 to show that $\bar{u}$ almost surely has a very specific structure.

Proposition 5. Let $b_{k}$ be as in Equation (27), $c_{k}$ as in Equation (28), $\bar{u}$ be as in Corollary 1 , and $\bar{u}_{k}:=\left\langle b_{k}, \bar{u}\right\rangle$. Then for $k<n$ and for almost all $\omega \in \Omega$

$$
\left(\frac{\bar{u}_{k}(\omega)}{c_{k}}\right)^{(n / k)^{2}} \leq \frac{\bar{u}_{n}(\omega)}{c_{n}} \leq 1-\left(1-\frac{\bar{u}_{k}(\omega)}{c_{k}}\right)^{(n / k)^{2}} .
$$

Proof. For each $k, n \in \mathbb{N}$, let $R_{k, n}$ be the closed planar region enclosed by the following two planar curves:

$$
\left\{P_{k, n}\left(e^{-B t} c\right): t \geq 0\right\} \quad \text { and } \quad\left\{P_{k, n}\left(c-e^{-B t} c\right): t \geq 0\right\} .
$$

Define $S_{k, n} \subset L^{2}[0, L]$ by

$$
S_{k, n}=\left\{f \in L^{2}[0, L]: P_{k, n}(f) \in R_{k, n}\right\} .
$$

It is straightforward to check that $S_{k, n}$ is invariant under $\Phi_{t}^{0}$ and $\Phi_{t}^{1}$ defined in Equation (??) for each $k, n \in \mathbb{N}$. Hence, $\cap_{k, n} S_{k, n}$ is invariant under $\Phi_{t}^{0}$ and $\Phi_{t}^{1}$ and we have by Proposition 3 that $Y_{0}$ and $Y_{1}$ (and hence $\bar{u}$ by Corollary 1) are almost surely contained in $\cap_{k, n} S_{k, n}$.

For $k<n$, observe that $R_{k, n}$ can be written as

$$
R_{k, n}=\left\{(x, y) \in \mathbb{R}^{2}: 0 \leq \frac{x}{c_{k}} \leq 1 \text { and }\left(\frac{x}{c_{k}}\right)^{(n / k)^{2}} \leq \frac{y}{c_{n}} \leq 1-\left(1-\frac{x}{c_{k}}\right)^{(n / k)^{2}}\right\} .
$$

The desired result follows. 
Furthermore, we have the following regularity result on $\bar{u}$. Notice that it implies that as we move to finer and finer spatial scales by take $k \rightarrow \infty$, there is asymptotically only one piece of randomness which determines the fine scale structure asymptotically.

Proposition 6. Let $r<1 / 2, b_{k}$ be as in Equation (27), $c_{k}$ as in Equation (28), $Y_{0}^{k}:=\left\langle b_{k}, Y_{0}\right\rangle$, and $Y_{1}^{k}:=\left\langle b_{k}, Y_{1}\right\rangle$. Then for each $\omega \in \Omega$, there exists an $M(\omega)$ so that

$$
1-\frac{M(\omega)}{k^{r}} \leq \frac{Y_{0}^{k}(\omega)}{c_{k}} \leq 1+\frac{M(\omega)}{k^{r}} \quad \text { and } \quad-\frac{M(\omega)}{k^{r}} \leq \frac{Y_{1}^{k}(\omega)}{c_{k}} \leq \frac{M(\omega)}{k^{r}}
$$

Proof. For each $k$, define

$$
A_{k}:=\left\{\omega \in \Omega:\left|\frac{Y_{0}^{k}(\omega)}{c_{k}}-\mathbb{E} \frac{Y_{0}^{k}}{c_{k}}\right|>\frac{1}{k^{r}}\right\} .
$$

By Chebyshev's inequality and Equation (29), we have that

$$
\mathbb{P}\left(A_{k}\right) \leq \frac{\operatorname{Var}\left(Y_{0}^{k}\right)}{c_{k}^{2}} k^{2 r}=\frac{\beta_{k} r_{0}\left(\beta_{k}+r_{1}\right)}{\left(\beta_{k}+r_{0}+r_{1}\right)^{2}\left(2 \beta_{k}+r_{0}+r_{1}\right)} k^{2 r} \sim k^{2(r-1)} \text { as } k \rightarrow \infty .
$$

Thus if $r<1 / 2$, then $\sum_{k=1}^{\infty} \mathbb{P}\left(A_{k}\right)<\infty$ and we conclude by the Borel-Cantelli Lemma that $\mathbb{P}\left(A_{k}\right.$ infinitely often $)=0$. Hence, for almost all $\omega \in \Omega$, we can choose an $M(\omega)$ so that for all $k$,

$$
\frac{r_{1}+\beta_{k}}{r_{0}+r_{1}+\beta_{k}}-\frac{M(\omega)}{k^{r}} \leq \frac{Y_{0}^{k}(\omega)}{c_{k}} \leq \frac{r_{1}+\beta_{k}}{r_{0}+r_{1}+\beta_{k}}+\frac{M(\omega)}{k^{r}} .
$$

A similar argument shows that for almost all $\omega \in \Omega$, we can choose an $M(\omega)$ so that for all $k$,

$$
\frac{r_{1}}{r_{0}+r_{1}+\beta_{k}}-\frac{M(\omega)}{k^{r}} \leq \frac{Y_{1}^{k}(\omega)}{c_{k}} \leq \frac{r_{1}}{r_{0}+r_{1}+\beta_{k}}+\frac{M(\omega)}{k^{r}}
$$

Since $\beta_{k} \sim k^{2}$ as $k \rightarrow \infty$, the desired results follows.

We can iterate this proposition to obtain the following result which shows that $Y_{0}^{k}$ and $Y_{1}^{k}$ depend essentially on only one switching time for large $k$. Note that we could continue to iterate this proposition to obtain similar bounds. Recall the definition of each $\omega \in \Omega$ in Equation (1).

Corollary 2. Let $r<1 / 2, b_{k}$ be as in Equation (27), $c_{k}$ as in Equation (28), $Y_{0}^{k}:=$ $\left\langle b_{k}, Y_{0}\right\rangle$, and $Y_{1}^{k}:=\left\langle b_{k}, Y_{1}\right\rangle$. Then for each $\omega \in \Omega$, there exists an $M_{0}(\omega)$ depending only on $\left\{\left(\tau_{0}^{k+1}, \tau_{1}^{k}\right)\right\}_{k \geq 1}$ and an an $M_{1}(\omega)$ depending only on $\left\{\left(\tau_{0}^{k}, \tau_{1}^{k+1}\right)\right\}_{k \geq 1}$ such that

$$
\begin{aligned}
1-e^{-\beta_{k} \tau_{0}^{1}}\left(\frac{M_{0}(\omega)}{k^{r}}+1\right) & \leq \frac{Y_{0}^{k}(\omega)}{c_{k}} \leq 1+e^{-\beta_{k} \tau_{0}^{1}}\left(\frac{M_{0}(\omega)}{k^{r}}-1\right) \\
e^{-\beta_{k} \tau_{1}^{1}}\left(1-\frac{M_{1}(\omega)}{k^{r}}\right) & \leq \frac{Y_{1}^{k}(\omega)}{c_{k}} \leq e^{-\beta_{k} \tau_{1}^{1}}\left(1+\frac{M_{1}(\omega)}{k^{r}}\right)
\end{aligned}
$$

Proof. Let $\omega$ be given. Define $\sigma: \omega \rightarrow \omega$ by

$$
\sigma(\omega)=\left(\left(\tau_{0}^{2}, \tau_{1}^{1}\right),\left(\tau_{0}^{3}, \tau_{1}^{2}\right),\left(\tau_{0}^{4}, \tau_{1}^{3}\right), \ldots\right)
$$

Then by the definition of $Y_{0}^{k}$ and $Y_{1}^{k}$, we have that

$$
\frac{Y_{0}^{k}(\omega)}{c_{k}}=1+e^{-\beta_{k} \tau_{0}^{1}}\left(\frac{Y_{1}^{k}(\sigma(\omega))}{c_{k}}-1\right)
$$


By Proposition 6, there exists an $M(\sigma(\omega))$ so that

$$
-\frac{M(\sigma(\omega))}{k^{r}} \leq \frac{Y_{1}^{k}(\sigma(\omega))}{c_{k}} \leq \frac{M(\sigma(\omega))}{k^{r}} .
$$

Thus,

$$
1-e^{-\beta_{k} \tau_{0}^{1}}\left(\frac{M(\sigma(\omega))}{k^{r}}+1\right) \leq \frac{Y_{0}^{k}(\omega)}{c_{k}} \leq 1+e^{-\beta_{k} \tau_{0}^{1}}\left(\frac{M(\sigma(\omega))}{k^{r}}-1\right) .
$$

The bounds on $Y_{1}^{k}$ are proved in a similar way.

4.3. Application to insect physiology. Essentially all insects breathe via a network of tubes that allows oxygen and carbon dioxide to diffuse to and from their cells [40. Air enters and exits this network through valve-like holes (called spiracles) in the exoskeleton. These spiracles regulate air flow by opening and closing. Surprisingly, spiracles have three distinct phases of activity, each typically lasting for hours. There is a completely closed phase, a completely open phase, and a flutter phase in which the spiracles rapidly open and close [27.

Insect physiologists have proposed at least five major hypotheses to explain the purpose of this behavior [11. In order to address these competing hypotheses, physiologists would like to understand how much cellular oxygen uptake decreases as a result of the spiracles closing.

To answer this question, we consider the following model. We represent a tube by the interval $[0, L]$ and model the oxygen concentration at a point $x \in[0, L]$ at time $t$ by the function $u(x, t)$. As diffusion is the primary mechanism for oxygen movement in the tubes (see 29]), the function $u$ satisfies the heat equation with some diffusion coefficient $D$. We impose an absorbing boundary condition at the left endpoint of the interval to represent cellular oxygen absorption where the tube meets the insect tissue. The right endpoint represents the spiracle, and since the spiracle opens and closes, the boundary condition here switches between a no flux boundary condition, $u_{x}(L, t)=0$ (spiracle closed) and a Dirichlet boundary condition, $u(L, t)=b>0$ (spiracle open). We suppose that the spiracle switches from open to closed and from closed to open with exponential rates $r_{0}$ and $r_{1}$ respectively.

Then, the oxygen concentration $u(x, t)$ is the same process described above in Secion 4.1. Using the results from that section, if we let $\rho=r_{0} / r_{1}$ and $\gamma=$ $L \sqrt{\left(r_{0}+r_{1}\right) / D}$, then the expected oxygen flux to the cells at large time is given by

$$
\left(1+\frac{\rho}{\gamma} \tanh (\gamma)\right)^{-1} \frac{b D}{L}
$$

This formula is noteworthy because it shows that the cellular oxygen uptake not only depends on the average proportion of time the spiracle is open, but it also depends on the overall rate of opening and closing. In particular, note that if we keep the ratio $\rho$ fixed, but let $\gamma$ become large, then the oxygen uptake approaches $\frac{b D}{L}$. In biological terms, this implies that the insect can have its spiracles open an arbitrarily small proportion of time, and yet receive essentially just as much oxygen as if its spiracles were always open if it opens and closes with a sufficiently high frequency.

Acknowledgements. JCM would like to thank Yuri Bakhtin for stimulating discussions. This research was partially supported by NSF grants EF-1038593 
(HFN, MCR), DMS-0854879 (JCM), DMS-0943760 (MCR), and NIH grant R01 ES019876 (DT).

\section{REFERENCES}

[1] Y. Bakhtin, Burgers equation with random boundary conditions, Proc. Amer. Math. Soc., 135 (2007), p. 22572262.

[2] Y. Bakhtin And T. Hurth, Invariant densities for dynamical systems with random switching, Nonlinearity, 25 (2012).

[3] M. Balde, U. Boscain, and P. Mason, A note on stability conditions for planar switched systems, Internat. J. Control, 82 (2009), pp. 1882-1888.

[4] I. Belykh, V. Belykh, R. Jeter, And M. Hasler, Multistable randomly switching oscillators: the odds of meeting a ghost, Eur. Phys. J. Spec. Top., (2013).

[5] M. Benaim, S. Leborgne, F. Malrieu, and P.A. Zitt, Qualitative properties of certain piecewise deterministic markov processes, preprint, (2012).

[6] M. Benaim, S. Leborgne, F. Malrieu, and P. A. Zitt, Quantitative ergodicity for some switched dynamical systems, Electron. Commun. Probab., 17 (2012), pp. 1-14.

[7] P. Billingsley, Convergence of Probability Measures, Wiley, Hoboken, 2nd ed., 1999.

[8] O. Boxma, H. Kaspi, O. Kella, And D. Perry, On/off storage systems with state-dependent input, output, and switching rates, Probab. Engrg. Inform. Sci, 19 (2005).

[9] P. Bressloff And J. Newby, Metastability in a stochastic neural network modeled as a velocity jump markov process, SIAM J. Appl. Dyn. Syst., 12 (2013), pp. 1394-1435.

[10] E. BUCKWAR AND M. RiedLeR, An exact stochastic hybrid model of excitable membranes including spatio-temporal evolution, J. Math. Biol., 63 (2011), pp. 1051-1093.

[11] S. Chown, A. Gibbs, S. Hetz, C. Klok, John R. Lighton, and E. Marais, Discontinuous gas exchange in insects: A clarification of hypotheses and approaches, Physiol. Biochem. Zool., 79 (2006), pp. 333-343.

[12] B. Cloez And M. HAirer, Exponential ergodicity for markov processes with random switching, Bernoulli, (to appear).

[13] H. Crauel, Random point attractors versus random set attractors, J. London Math. Soc. (2), 63 (2001), pp. 413-427.

[14] H. Crauel and F. Flandoli, Attractors for random dynamical systems, Probab. Theory Related Fields, 100 (1994), pp. 365-393.

[15] G. Da Prato AND J. ZABCZYK, Evolution equations with white-noise boundary conditions, Stoch. and Stoch. Rep., 42 (1993), pp. 431-459.

[16] P. Diaconis And D. Freedman, Iterated random functions, SIAM Rev., 41 (1999), p. 4576.

[17] J. Duan, S. Luo, And C. Wang, Hyperbolic equations with random boundary conditions, in Recent development in Stochastic Dynamics and Stochastic Analysis, vol. 8, World Scientific, 2010.

[18] M. Duflo, Random iterative models, vol. 34 of Applications of Mathematics (New York), Springer-Verlag, Berlin, 1997. Translated from the 1990 French original by Stephen S. Wilson and revised by the author.

[19] R. Durrett, Probability : Theory and Examples, Cambridge University Press, Cambridge, 4th ed., 2010.

[20] J. Farkas, P. Hinow, and J. Engelstadter, Pathogen evolution in switching environments: A hybrid dynamical system approach, Math. Biosci., 240 (2012), pp. 70-75.

[21] M. Hasler, V. Belykh, and I. Belykh, Dynamics of stochastically blinking systems. part $i$ : Finite time properties, SIAM J. Appl. Dyn. Syst., 12 (2013), pp. 1007-1030.

[22] — Dynamics of stochastically blinking systems. part ii: Asymptotic properties, SIAM J. Appl. Dyn. Syst., 12 (2013), pp. 1031-1084.

[23] T. HuRTh, Limit theorems for a one-dimensional system with random switchings, master's thesis, Georgia Institute of Technology, December 2010.

[24] Y. Kifer, Ergodic theory of random transformations, vol. 10 of Progress in Probability and Statistics, Birkhäuser Boston, Inc., Boston, MA, 1986.

[25] —, Random perturbations of dynamical systems, vol. 16 of Progress in Probability and Statistics, Birkhäuser Boston, Inc., Boston, MA, 1988.

[26] S. D. Lawley, J. C. Mattingly, and M. C. Reed, Sensitivity to switching rates in stochastically switched ODEs, Commun. Math. Sci., 12 (2014), pp. 1343-1352. 
[27] J. Lighton, Discontinuous gas exchange in insects, Ann. Rev. of Ent., 41 (1996), pp. 309324. PMID: 8546448.

[28] H. Lin And P. J. Antsaklis, Stability and stabilizability of switched linear systems: A survey of recent results, IEEE Trans. Automat. Contr., 54 (2009).

[29] C. Loudon, Tracheal hypertrophy in mealworms: Design and plasticity in oxygen supply systems, J. Exp. Bio., 147 (1989), pp. 217-235.

[30] J. C. Mattingly, Ergodicity of $2 D$ Navier-Stokes equations with random forcing and large viscosity, Comm. Math. Phys., 206 (1999), pp. 273-288.

[31] - Contractivity and ergodicity of the random map $x \mapsto|x-\theta|$, Teor. Veroyatnost. i Primenen., 47 (2002), pp. 388-397.

[32] J. C. Mattingly And T. M. Suidan, The small scales of the stochastic Navier-Stokes equations under rough forcing, J. Stat. Phys., 118 (2005), pp. 343-364.

[33] - Transition measures for the stochastic Burgers equation, in Integrable systems and random matrices, vol. 458 of Contemp. Math., Amer. Math. Soc., Providence, RI, 2008, pp. 409-418.

[34] J. Newby And J. KeEner, An asymptotic analysis of the spatially inhomogeneous velocityjump process, Multiscale Model. Simul., 9 (2011), pp. 735-765.

[35] S. M. Ross, Stochastic processes, Wiley, New York, 2nd ed., 1996.

[36] B. Schmalfuss, A random fixed point theorem based on Lyapunov exponents, Random Comput. Dynam., 4 (1996), pp. 257-268.

[37] R. Schnaubelt And M. VeraAr, Stochastic equations with boundary noise, in Parabolic Problems, J. Escher, P. Guidotti, M. Hieber, P. Mucha, J. Pruss, Y. Shibata, G. Simonett, C. Walker, and W. Zajaczkowski, eds., vol. 80 of Progr. Nonlinear Differential Equations Appl., Springer Basel, 2011, pp. 609-629.

[38] W. WANG AND J. DUAN, Reductions and deviations for stochastic partial differential equations under fast dynamical boundary conditions, Stoch. Anal. Appl., 27 (2009), p. 431459.

[39] E. Weinan, K. Khanin, A. Mazel, And Y. Sinai, Invariant measures for Burgers equation with stochastic forcing, Ann. of Math. (2), 151 (2000), pp. 877-960.

[40] V. B. Wigglesworth, The respiration of insects, Bio. Rev., 6 (1931), p. 181220.

[41] InC. Wolfram Research, Mathematica, 2012.

[42] G. Yin AND C. ZHU, Hybrid Switching Diffusions, Springer, New York, USA, 2010.

[43] C. Zhu ANd G. Yin, On competitive Lotka Volterra model in random environments, J. Math. Anal. Appl., 357 (2009), pp. 154-170. 